\title{
Boundary Control Problem for Heat Convection Equations with Slip Boundary Condition
}

\author{
Exequiel Mallea-Zepeda $\left(\mathbb{D},{ }^{1}\right.$ Eber Lenes, ${ }^{2}$ and Elvis Valero ${ }^{1}$ \\ ${ }^{1}$ Departamento de Matemática, Universidad de Tarapacá, Av. 18 de Septiembre, 2222 Arica, Chile \\ ${ }^{2}$ Departamento de Investigaciones, Universidad del Sinú, Elías Bechara Zainúm, Av. al Bosque Tr. 54 No. 30-729, Cartagena, Colombia \\ Correspondence should be addressed to Exequiel Mallea-Zepeda; emallea@uta.cl
}

Received 4 October 2017; Accepted 16 December 2017; Published 28 January 2018

Academic Editor: Qin Yuming

Copyright (c) 2018 Exequiel Mallea-Zepeda et al. This is an open access article distributed under the Creative Commons Attribution License, which permits unrestricted use, distribution, and reproduction in any medium, provided the original work is properly cited.

\begin{abstract}
We analyze an optimal boundary control problem for heat convection equations in a three-dimensional domain, with mixed boundary conditions. We prove the existence of optimal solutions, by considering boundary controls for the velocity vector and the temperature. The analyzed optimal control problem includes the minimization of a Lebesgue norm between the velocity and some desired field, as well as the temperature and some desired temperature. By using the Lagrange multipliers theorem we derive an optimality system. We also give a second-order sufficient condition.
\end{abstract}

\section{Introduction}

Let $\Omega \subset \mathbb{R}^{3}$ be a simply connected bounded domain with Lipschitz boundary $\Gamma$. We deal with the existence of weak solutions of a boundary value problem describing the motion of a viscous heat conduction fluid in $\Omega$, with a Navier slip condition on the boundary for the velocity vector. This model is given by the following system of partial differential equations:

$$
\begin{array}{rlrl}
-v \Delta \mathbf{u}+(\mathbf{u} \cdot \nabla) \mathbf{u}+\nabla p+\beta \mathbf{G} T & =\mathbf{f} & & \text { in } \Omega, \\
-\kappa \Delta T+\mathbf{u} \cdot \nabla T & =g & & \text { in } \Omega, \\
\operatorname{div} \mathbf{u}=0 & & \text { in } \Omega .
\end{array}
$$

Here, $\mathbf{u}(x)$ is the velocity field, $p(x)$ represents the hydrostatic pressure, and $T(x)$ is the temperature of the fluid at point $x \in \Omega$; the constant $\nu>0$ denotes the kinematic viscosity; $\beta$ is the volume extension coefficient; $\mathbf{G}(x)$ is the free fall acceleration vector; the field $\mathbf{f}(x)$ represents external source of linear momentum; the constant $\kappa>0$ is the temperature conduction and $g(x)$ is the volume density of heat sources. Without loss of generality, we assume that density of the fluid is equal to one; thus if the variables in (1)-(2) are nondimensionalized, then the coefficient of viscosity $v$ is simply the inverse of the Reynolds number Re (see, for example, [1] p. 166 and [2]).

We prove the existence of weak solutions for system (1)-(3). Moreover, we study an optimal boundary control problem. To do this, we consider the following boundary conditions:

$$
\begin{aligned}
\mathbf{u}_{\mathrm{g}_{1}} & = \begin{cases}\mathbf{u}_{0} & \text { on } \Gamma_{1}, \\
\mathbf{g}_{1} & \text { on } \Gamma_{2},\end{cases} \\
\mathbf{u} \cdot \mathbf{n} & =0, \\
{[D(\mathbf{u}) \mathbf{n}+\alpha \mathbf{u}]_{\operatorname{tang}} } & =0 \quad \text { on } \Gamma_{3}, \\
T & =g_{2} \quad \text { on } \Gamma_{4}, \\
\kappa\left(\frac{\partial T}{\partial \mathbf{n}}+\gamma T\right) & =\varphi \quad \text { on } \Gamma_{5} .
\end{aligned}
$$

Here the boundary $\Gamma=\Gamma_{1} \cup \Gamma_{2} \cup \Gamma_{3}=\Gamma_{4} \cup \Gamma_{5}$, where $\bar{\Gamma}_{1} \cap \bar{\Gamma}_{2}=\bar{\Gamma}_{1} \cap \bar{\Gamma}_{3}=\bar{\Gamma}_{2} \cap \bar{\Gamma}_{3}=\bar{\Gamma}_{4} \cap \bar{\Gamma}_{5}=\emptyset$, and $\mathbf{n}$ denotes the outward normal vector on $\Gamma$. The function $\mathbf{u}_{0}$ is defined on $\Gamma_{1} ; \gamma \geq 0$ and $\varphi$ are functions givens on $\Gamma_{5}$, and the functions $\mathbf{g}_{1}, g_{2}$ describe the Dirichlet boundary control for velocity $\mathbf{u}$ on $\Gamma_{2}$ and for temperature $T$ on $\Gamma_{4}$, respectively.The controls 
$\mathbf{g}_{1}, g_{2}$ lie in the closed convex sets $\mathcal{U}_{1} \subset \mathbf{H}^{1 / 2}\left(\Gamma_{2}\right)$ and $\mathcal{U}_{2} \subset$ $H^{1 / 2}\left(\Gamma_{4}\right)$, respectively. The term $[D(\mathbf{u}) \mathbf{n}+\alpha \mathbf{u}]_{\operatorname{tang}}:=D(\mathbf{u}) \mathbf{n}+$ $\alpha \mathbf{u}-[(D(\mathbf{u}) \mathbf{n}+\alpha \mathbf{u}) \cdot \mathbf{n}] \mathbf{n}$ represents the tangential component of the vector $D(\mathbf{u}) \mathbf{n}+\alpha \mathbf{u}$, where $D(\mathbf{u}):=(1 / 2)\left(\nabla \mathbf{u}+\nabla^{T} \mathbf{u}\right)$ is the deformation tensor, and the real number $\alpha \geq 0$ is the friction coefficient which measures the tendency of the fluid to slip on $\Gamma_{3}$. When $\alpha=0$ and the boundary is flat, the fluid slips along the $\Gamma_{3}$ without friction and there is no boundary layers. When $\alpha \rightarrow+\infty$, the friction is so intense that the fluid is almost at rest near the boundary; the condition $[D(\mathbf{u}) \mathbf{n}+\alpha \mathbf{u}]_{\text {tang }}=$ $\mathbf{0}, \mathbf{u} \cdot \mathbf{n}=0$ on $\Gamma_{3}$ converges to the Dirichlet condition. The case when $\alpha$ is a scalar function has been studied in [3], for the 2D nonstationary Navier-Stokes equations. The condition $[D(\mathbf{u}) \mathbf{n}+\alpha \mathbf{u}]_{\operatorname{tang}}=\mathbf{0}, \mathbf{u} \cdot \mathbf{n}=0$ on $\Gamma_{3}$ is a Navier friction boundary condition. The Navier boundary condition was proposed by Navier [4], who claimed that the tangential component of the viscous stress at the boundary should be proportional to the tangential velocity. Navier boundary condition was also derived by Maxwell [5] from the kinetic theory of gases and rigorously justified as a homogenization of the no-slip condition on a rough boundary [6].

Before we describe in detail our results, let us briefly comment on previous related work. The heat convection equations, or also known as Boussinesq model, have been extensively analyzed, and, among the many articles on these equations, we just mention [7-12]. In these papers are studied the existence of very weak, weak, and strong solutions and uniqueness with Dirichlet boundary conditions on the velocity and temperature or Dirichlet boundary condition on the velocity and mixed boundary conditions on the temperature. The tools used are Galerkin method, linearization, and fixed point results. The results obtained are similar to those known for the classical Navier-Stokes equations.

When Navier friction boundary condition is considered, the authors do not know results of existence for the classical Boussinesq model, except work due to Rionero and Mulone [13], where it is considered a slightly more general model on thermodiffusive mixture.

In the context of the classic Navier-Stokes equations there is a good amount of work with Navier friction boundary condition, among which we can mention [3,14-19], and references therein, where the existence, regularity, and uniqueness of weak and strong solutions are studied. In the context of steady-state microstructure fluids, also called micropolar fluids, we can mention works $[20,21]$. In [20] a threedimensional domain is considered where the density of the fluid is constant, while in [21] the study is performed on a two-dimensional domain with variable density.

For optimal control problems for the classical Boussinesq stationary model, see [22-27], and the references therein. In such works, standard results have been obtained, such as the existence of an optimal solution and stability as well as first-order necessary optimality conditions, from which the authors derive an optimality system. However, as far as we know, for this model there are no known studies of sufficient second-order conditions for the existence of a local extreme, except for the recent work [28], in which the authors study a problem of control of limits associated with the stationary system of Rayleigh-Bénard-Marangoni (similar to system (1)-(3)), for which they obtain results of existence, uniqueness, and regularity of the model; in addition they provide the existence of optimal solutions, derive optimality conditions, obtain a second-order sufficient condition, and establish a result of uniqueness for the optimal solution.

The outline of this paper is as follows. In Section 2, we introduce the spaces of functions appropriate for the development of the article. In Section 3, we prove the existence and uniqueness of weak solutions to model (1)-(4), by using the Galerkin method. In Section 4, we establish the optimal control problem and also we prove the existence of optimal solutions, and, using the Lagrange multipliers method, we obtain the first-order optimality conditions, from which we derive an optimality system. In Section 5, we obtain a second-order sufficient optimality condition.

\section{Preliminaries}

Throughout this paper we will use the classical Lebesgue space $L^{p}(\Omega), 1 \leq p \leq \infty$, with norm denoted by $\|\cdot\|_{p}$. In particular, the norm and inner product in $L^{2}(\Omega)$ will be represented by $\|\cdot\|$ and $(\cdot, \cdot)$, respectively. The norm of $L^{p}(\Gamma)$ will be denoted by $\|\cdot\|_{L^{p}(\Gamma)}$. Also we use the Sobolev space $H^{1}(\Omega):=W^{1,2}(\Omega)=\left\{u \in L^{2}(\Omega): D^{\alpha} u \in L^{2}(\Omega)\right\}$, for a multi-index $\alpha$, with $|\alpha|=1$. Its norm will be denoted by $\|\cdot\|_{H^{1}}$. Spaces of $\mathbb{R}^{3}$ valued functions, as well as their elements, are represented by bold face letters; thus, for example, we have $\mathbf{H}^{1}(\Omega), \mathbf{L}^{2}(\Omega)$, and so on. We will use the Hilbert space $H_{0}=\left\{T \in H^{1}(\Omega): T=0\right.$ on $\left.\Gamma_{4}\right\}$ with the inner product $(u, v)_{H_{0}}:=(\nabla u, \nabla v)$ and norm $\|u\|_{H_{0}}:=\|\nabla u\|$. We also consider the following solenoidal Banach spaces $\mathbf{H}_{\sigma}^{1}=\{\mathbf{u} \epsilon$ $\mathbf{H}^{1}(\Omega): \operatorname{div} \mathbf{u}=0$ and $\mathbf{u} \cdot \mathbf{n}=0$ on $\left.\Gamma_{3}\right\}$, endowed with the usual norm of $\mathbf{H}^{1}(\Omega)$; the space $\widetilde{\mathbf{H}}_{\sigma}^{1}=\left\{\mathbf{u} \in \mathbf{H}_{\sigma}^{1}: \mathbf{u}=\right.$ $\mathbf{0}$ on $\Gamma \backslash \Gamma_{3}$, which is a Hilbert space with the inner product $(\mathbf{u}, \mathbf{v})_{\widetilde{\mathbf{H}}_{\sigma}^{1}}:=(D(\mathbf{u}), D(\mathbf{v}))$ and norm $\|\mathbf{u}\|_{\widetilde{\mathbf{H}}_{\sigma}^{1}}:=\|D(\mathbf{u})\|$.

If $X$ is general Banach space, its topological dual will be denoted by $X^{\prime}$ and the duality product by $\langle\cdot, \cdot\rangle_{X^{\prime}}$ or simply by $\langle\cdot, \cdot\rangle$ unless this leads to ambiguity. The space $H^{\prime}$ denotes the dual of $H_{0}$, the space $\mathbf{H}_{\sigma}^{\prime}$ denotes the dual of $\mathbf{H}_{\sigma}^{1}$, and the space $\widetilde{\mathbf{H}}_{\sigma}^{\prime}$ denotes the dual of $\widetilde{\mathbf{H}}_{\sigma}^{1}$. For simplicity, we consider the spaces $\mathbb{X}=\widetilde{\mathbf{H}}_{\sigma}^{1} \times H_{0}, \mathbb{X}^{\prime}=\widetilde{\mathbf{H}}_{\sigma}^{\prime} \times H^{\prime}$. If $\Gamma_{k}$ is a connected subset of $\Gamma$, we consider the trace space by $\mathbf{H}^{1 / 2}\left(\Gamma_{k}\right)=\left\{\mathbf{u}_{\mid \Gamma_{k}}\right.$ : $\left.\mathbf{u} \in \mathbf{H}^{1}(\Omega)\right\}$ (the restriction of the elements of $\mathbf{H}^{1}(\Omega)$ to $\Gamma_{k}$ ) and

$$
\begin{aligned}
& \mathbf{H}_{00}^{1 / 2}\left(\Gamma_{k}\right)=\left\{\mathbf{v} \in \mathbf{L}^{2}\left(\Gamma_{k}\right): \text { there exists } \widehat{\mathbf{v}}\right. \\
& \left.\quad \in \mathbf{H}^{1 / 2}(\Gamma), \widehat{\mathbf{v}}_{|\Gamma| \Gamma_{k}}=\mathbf{0}, \widehat{\mathbf{v}}_{\mid \Gamma_{k}}=\mathbf{v}\right\}, \\
& \widetilde{\mathbf{H}}_{00}^{1 / 2}\left(\Gamma_{k}\right)=\left\{\mathbf{v} \in \mathbf{H}_{00}^{1 / 2}\left(\Gamma_{k}\right): \int_{\Gamma_{k}} \mathbf{v} \cdot \mathbf{n} d \Gamma=0\right\} .
\end{aligned}
$$

The space $\mathbf{H}_{00}^{1 / 2}\left(\Gamma_{k}\right)$ is a closed subspace of $\mathbf{H}^{1 / 2}\left(\Gamma_{k}\right)$; moreover, it is continuously embedded in $\mathbf{L}^{2}\left(\Gamma_{k}\right)$ (see $\left.[29,30]\right)$. For the space $\mathbf{H}^{1 / 2}\left(\Gamma_{k}\right), \mathbf{H}^{-1 / 2}\left(\Gamma_{k}\right)$ denote its dual space and $\langle\cdot, \cdot\rangle_{\Gamma_{k}}$ denote the duality. The letter $C$ will denote diverse positive 
constants which may change from line to line or even within the same line.

In order to establish the weak formulation of problem (1)-(4) we consider the following result.

Lemma $\mathbf{1}$ (see [19]). Let $\mathbf{u} \in \mathbf{H}^{2}(\Omega)$ and $\mathbf{v} \in \mathbf{H}^{1}(\Omega)$ be divergence-free vector fields tangent to the boundary $\Gamma$. Then,

$$
\begin{aligned}
-\int_{\Omega} \Delta \mathbf{u} \cdot \mathbf{v} d x= & 2 \int_{\Omega} D(\mathbf{u}): D(\mathbf{v}) d x \\
& -2 \int_{\Gamma}[D(\mathbf{u}) \mathbf{n}]_{\operatorname{tang}} \cdot \mathbf{v} d \Gamma
\end{aligned}
$$

Then, motivated by the formula of integration by parts and Lemma 1, we establish the following definition of weak solution of system (1)-(4).

Definition 2. Let $(\mathbf{f}, g) \in \mathbb{X}^{\prime}, \mathbf{G} \in \mathbf{L}^{\infty}(\Omega), \mathbf{u}_{0} \in \widetilde{\mathbf{H}}_{00}^{1 / 2}\left(\Gamma_{1}\right), \mathbf{g}_{1} \in$ $\widetilde{\mathbf{H}}_{00}^{1 / 2}\left(\Gamma_{2}\right), g_{2} \in H_{00}^{1 / 2}\left(\Gamma_{4}\right), \varphi \in L^{2}\left(\Gamma_{5}\right)$, and $\gamma \in L^{\infty}\left(\Gamma_{5}\right)$. A weak solution of (1)-(4) is a pair $(\mathbf{u}, T) \in \mathbf{H}_{\sigma}^{1} \times H^{1}(\Omega)$ satisfying

$$
\begin{aligned}
& 2 \nu(D(\mathbf{u}), D(\mathbf{v}))+2 \alpha \nu \int_{\Gamma_{3}} \mathbf{u} \cdot \mathbf{v} d \Gamma+(\mathbf{u} \cdot \nabla \mathbf{u}, \mathbf{v}) \\
& \quad+\beta(\mathbf{G} T, \mathbf{v})=\langle\mathbf{f}, \mathbf{v}\rangle_{\widetilde{\mathbf{H}}_{\sigma}^{\prime}}, \\
& \kappa(\nabla T, \nabla z)+(\mathbf{u} \cdot \nabla T, z)+\kappa \int_{\Gamma_{5}} \gamma T \cdot z d \Gamma=\langle g, z\rangle_{H^{\prime}} \\
& \quad+\langle\varphi, z\rangle_{\Gamma_{5}}, \\
& \mathbf{u}=\mathbf{u}_{\mathbf{g}_{1}} \text { on } \Gamma \backslash \Gamma_{3}, \\
& T=g_{2} \text { on } \Gamma_{4},
\end{aligned}
$$

for all $(\mathbf{v}, z) \in \mathbb{X}$.

\section{Existence of a Weak Solution to Problem (1)-(4)}

In order to prove the existence of a solution to system (7)-(9), we reduce the problem to an auxiliary problem with homogeneous conditions for the fields velocity on $\Gamma \backslash \Gamma_{3}$ and the temperature on $\Gamma_{4}$. For this, we introduce the following results.

Lemma 3. Assume that $\mathbf{u}_{0} \in \widetilde{\mathbf{H}}_{00}^{1 / 2}\left(\Gamma_{1}\right), \mathbf{g}_{1} \in \widetilde{\mathbf{H}}_{00}^{1 / 2}\left(\Gamma_{2}\right)$. For any $\varepsilon>0$ there exists $\mathbf{u}^{\varepsilon} \in \mathbf{H}_{\sigma}^{1}$ with $\mathbf{u}^{\varepsilon}=\mathbf{g}_{1}$ on $\Gamma_{2}, \mathbf{u}^{\varepsilon}=\mathbf{u}_{0}$ on $\Gamma_{1}$, and $\mathbf{u}^{\varepsilon}=\mathbf{0}$ on $\Gamma_{3}$ such that

$$
\begin{gathered}
\left|\left(\mathbf{v} \cdot \nabla \mathbf{u}^{\varepsilon}, \mathbf{v}\right)\right| \leq \varepsilon\|\mathbf{v}\|_{\widetilde{\mathbf{H}}_{\sigma}^{1}}^{2} \quad \forall \mathbf{v} \in \widetilde{\mathbf{H}}_{\sigma}^{1}, \\
\left\|\mathbf{u}^{\varepsilon}\right\|_{\mathbf{H}_{\sigma}^{1}} \leq C\left\|\mathbf{u}_{\mathbf{g}_{1}}\right\|_{\mathbf{H}^{1 / 2}\left(\Gamma \backslash \Gamma_{3}\right)},
\end{gathered}
$$

where the constant $C>0$ depends only on $\Omega$. Moreover, if $g_{2} \epsilon$ $H_{00}^{1 / 2}\left(\Gamma_{4}\right)$, then there exists $T^{\delta} \in H^{1}(\Omega)$ such that

$$
\begin{aligned}
T^{\delta} & =g_{2} \quad \text { on } \Gamma_{4}, \\
\frac{\partial T^{\delta}}{\partial n}+\gamma T^{\delta} & =0 \quad \text { on } \Gamma_{5}, \\
\left\|T^{\delta}\right\|_{4} & \leq \delta, \\
\left\|T^{\delta}\right\|_{H^{1}} & \leq C\left\|g_{2}\right\|_{H^{1 / 2}\left(\Gamma_{4}\right)},
\end{aligned}
$$

where the constant $C$ depends only on $\Omega$.

Proof. The existence of $\mathbf{u}^{\varepsilon} \in \mathbf{H}_{\sigma}^{1}$, satisfying the relations given in (10), follows from [21], Lemma 3 (see, also [20], Lemma 3.2). The existence of $T^{\delta} \in H^{1}(\Omega)$ satisfying (11) follows from [31].

Lemma 4 (see [32]). Let $X$ be a finite dimensional Hilbert space with inner product $(\cdot, \cdot)_{X}$ and norm $\|\cdot\|_{X}$, and let $P$ be a continuous mapping from $X$ into itself such that

$$
(P(\phi), \phi)_{X}>0 \text { for }\|\phi\|_{X}=K>0 \text {. }
$$

Then, there exists $\phi \in X,\|\phi\|_{X} \leq K$, such that $P(\phi)=0$.

Rewriting the unknowns $(\mathbf{u}, T) \in \mathbf{H}_{\sigma}^{1} \times H^{1}(\Omega)$ in the form $\mathbf{u}=\mathbf{u}^{\varepsilon}+\widehat{\mathbf{u}}$ and $T=T^{\delta}+\widehat{T}$, where $(\widehat{\mathbf{u}}, \widehat{T}) \in \mathbb{X}$ are new unknown functions, from (7)-(9) we can obtain the following problem. Find $(\widehat{\mathbf{u}}, \widehat{T}) \in \mathbb{X}$ such that

$$
\begin{aligned}
& 2 \nu(D(\widehat{\mathbf{u}}), D(\mathbf{v}))+2 \alpha \nu \int_{\Gamma_{3}} \widehat{\mathbf{u}} \cdot \mathbf{v} d \Gamma+(\widehat{\mathbf{u}} \cdot \nabla \widehat{\mathbf{u}}, \mathbf{v}) \\
& \quad+\left(\widehat{\mathbf{u}} \cdot \nabla \mathbf{u}^{\varepsilon}, \mathbf{v}\right)+\left(\mathbf{u}^{\varepsilon} \cdot \nabla \widehat{\mathbf{u}}, \mathbf{v}\right) \\
& \quad+\beta(\mathbf{G} \widehat{T}, \mathbf{v})=\langle\widehat{\mathbf{f}}, \mathbf{v}\rangle_{\widetilde{\mathbf{H}}_{\sigma}^{\prime}} \quad \forall \mathbf{v} \in \widetilde{\mathbf{H}}_{\sigma}^{1}, \\
& \kappa(\nabla \widehat{T}, \nabla z)+(\widehat{\mathbf{u}} \cdot \nabla \widehat{T}, z)+\left(\widehat{\mathbf{u}} \cdot \nabla T^{\delta}, z\right)+\left(\mathbf{u}^{\varepsilon} \cdot \nabla \widehat{T}, z\right) \\
& \quad+\kappa \int_{\Gamma_{5}} \gamma \widehat{T} \cdot z d \Gamma=\langle\widehat{g}, z\rangle_{H^{\prime}} \quad \forall z \in H_{0},
\end{aligned}
$$

where

$$
\begin{aligned}
\langle\widehat{\mathbf{f}}, \mathbf{v}\rangle_{\widetilde{\mathbf{H}}_{\sigma}^{\prime}}:= & -2 \nu\left(D\left(\mathbf{u}^{\varepsilon}\right), D(\mathbf{v})\right) \\
& -\left(\mathbf{u}^{\varepsilon} \cdot \nabla \mathbf{u}^{\varepsilon}+\beta \mathbf{G} T^{\delta}, \mathbf{v}\right)+\langle\mathbf{f}, \mathbf{v}\rangle_{\widetilde{\mathbf{H}}_{\sigma}^{\prime}}, \\
\langle\widehat{g}, z\rangle_{H^{\prime}}:= & -\kappa\left(\nabla T^{\delta}, \nabla z\right)-\left(\mathbf{u}^{\varepsilon} \cdot \nabla T^{\delta}, z\right) \\
& -\kappa \int_{\Gamma_{5}} \gamma T^{\delta} \cdot z d \Gamma+\langle g, z\rangle_{H^{\prime}} \\
& +\langle\varphi, z\rangle_{\Gamma_{5}} .
\end{aligned}
$$

Thus, we have the following result.

Theorem 5. Let $(\mathbf{f}, g) \in \mathbb{X}^{\prime}, \mathbf{G} \in \mathbf{L}^{\infty}(\Omega), \mathbf{u}_{0} \in \widetilde{\mathbf{H}}_{00}^{1 / 2}\left(\Gamma_{1}\right)$, $\mathbf{g}_{1} \in \widetilde{\mathbf{H}}_{00}^{1 / 2}\left(\Gamma_{2}\right), g_{2} \in H_{00}^{1 / 2}\left(\Gamma_{4}\right), \varphi \in L^{2}\left(\Gamma_{5}\right)$, and $\gamma \in L^{\infty}\left(\Gamma_{5}\right)$. 
Given these conditions, if $\nu$ is large enough and $\|\gamma\|_{L^{\infty}\left(\Gamma_{5}\right)}$ is small enough such that

$$
\min \left\{2 v, \kappa-\kappa C\|\gamma\|_{L^{\infty}\left(\Gamma_{5}\right)}\right\}>C\left(\beta\|\mathbf{G}\|_{\infty}+\delta\right),
$$

where $C$ is a positive constant depending on $\Omega$ such that $\|\nabla \mathbf{u}\| \leq$ $C\|\mathbf{u}\|_{\widetilde{\mathbf{H}}_{\sigma}^{1}}$ and $\delta$ is given in (11), then, there exists $(\widehat{\mathbf{u}}, \widehat{T}) \in \mathbb{X}$, which is a solution of system (13).

Moreover, the following estimate is satisfied:

$$
\|(\widehat{\mathbf{u}}, \widehat{T})\|_{\mathbb{X}} \leq \frac{1}{\widetilde{\delta}}\|(\widehat{\mathbf{f}}, \widehat{g})\|_{\mathbb{X}^{\prime}},
$$

where $\widetilde{\delta}:=\min \left\{2 \nu-\varepsilon, \kappa-\kappa C\|\gamma\|_{L^{\infty}\left(\Gamma_{5}\right)}\right\}-C\left(\beta\|\mathbf{G}\|_{\infty}+\delta\right)>0$.

Proof. The existence of a solution will be proved by using the Galerkin method. For this purpose, we consider an approximate solution of (13) and then we pass to the limit. Since $\widetilde{\mathbf{H}}_{\sigma}^{1}$ and $H_{0}$ are separable spaces, there exist Hilbert spaces bases $\left\{\mathbf{v}_{i}\right\}_{i=1}^{\infty}$ and $\left\{z_{i}\right\}_{i=1}^{\infty}$ of $\widetilde{\mathbf{H}}_{\sigma}^{1}$ and $H_{0}$, respectively. For each $k \in \mathbb{N}$, we consider $\widetilde{\mathbf{H}}_{k}$ the space spanned by $\left\{\mathbf{v}_{1}, \ldots, \mathbf{v}_{k}\right\}$ and $H_{k}$ the space spanned by $\left\{z_{1}, \ldots, z_{k}\right\}$; let $\mathbb{X}_{k}=\widetilde{\mathbf{H}}_{k} \times H_{k} \subset$ $\mathbb{X}$. The scalar product on $\mathbb{X}_{k}$ is the scalar product $(\cdot, \cdot)_{\mathbb{X}_{k}}$ induced by $\mathbb{X}$.

Thus, for each $k \in \mathbb{N}$, fixed, we define an approximate solution $\left(\mathbf{u}_{k}, T_{k}\right) \in \mathbb{X}_{k}$ of system (13), given by

$$
\begin{array}{r}
\mathbf{u}_{k}:=\sum_{i=1}^{k} \xi_{i, k} \mathbf{v}_{i}(x), \\
T_{k}(x):=\sum_{i=1}^{k} \theta_{i, k} T_{i}(x),
\end{array}
$$

where $\xi_{i, k}, \theta_{i, k} \in \mathbb{R}$, and satisfying

$$
\begin{aligned}
2 \nu & \left(D\left(\mathbf{u}_{k}\right), D\left(\mathbf{v}_{i}\right)\right)+2 \alpha \nu \int_{\Gamma_{3}} \mathbf{u}_{k} \cdot \mathbf{v}_{i} d \Gamma+\left(\mathbf{u}_{k} \cdot \nabla \mathbf{u}_{k}, \mathbf{v}_{i}\right) \\
& +\left(\mathbf{u}_{k} \cdot \nabla \mathbf{u}^{\varepsilon}, \mathbf{v}_{i}\right)+\left(\mathbf{u}^{\varepsilon} \cdot \nabla \mathbf{u}_{k}, \mathbf{v}_{i}\right) \\
& +\beta\left(\mathbf{G} T_{k}, \mathbf{v}_{i}\right)=\left\langle\widehat{\mathbf{f}}, \mathbf{v}_{i}\right\rangle_{\widetilde{\mathbf{H}}_{\sigma}^{\prime}}, \\
\kappa & \left(\nabla T_{k}, \nabla z_{i}\right)+\left(\mathbf{u}_{k} \cdot \nabla T_{k}, z_{i}\right)+\left(\mathbf{u}_{k} \cdot \nabla T^{\delta}, z_{i}\right) \\
& +\left(\mathbf{u}^{\varepsilon} \cdot \nabla T_{k}, z_{i}\right)+\kappa \int_{\Gamma_{5}} \gamma T_{k} \cdot z_{i} d \Gamma=\left\langle\widehat{g}, z_{i}\right\rangle_{H^{\prime}}
\end{aligned}
$$

for all $\left(\mathbf{v}_{i}, z_{i}\right) \in \mathbb{X}_{k}$.

In order to prove the existence of a solution for system (18) we apply the result of Lemma 4. For this purpose, we consider the continuous mapping $P: \mathbb{X}_{k} \rightarrow \mathbb{X}_{k}$, which for $\mathbf{w}_{k}=\left(\mathbf{u}_{k}, T_{k}\right) \in \mathbb{X}_{k}$ is given by

$$
\begin{aligned}
\left(P\left(\mathbf{w}_{k}\right), \mathbf{w}\right)_{\mathbb{X}_{k}}= & 2 \nu\left(D\left(\mathbf{u}_{k}\right), D(\mathbf{v})\right) \\
& +2 \alpha \nu \int_{\Gamma_{3}} \mathbf{u}_{k} \cdot \mathbf{v} d \Gamma+\left(\mathbf{u}_{k} \cdot \nabla \mathbf{u}_{k}, \mathbf{v}\right) \\
& +\left(\mathbf{u}_{k} \cdot \nabla \mathbf{u}^{\varepsilon}, \mathbf{v}\right)+\left(\mathbf{u}^{\varepsilon} \cdot \nabla \mathbf{u}_{k}, \mathbf{v}\right)
\end{aligned}
$$

$$
\begin{aligned}
& +\beta\left(\mathbf{G} T_{k}, \mathbf{v}\right)-\langle\widehat{\mathbf{f}}, \mathbf{v}\rangle_{\widetilde{\mathbf{H}}_{\sigma}^{\prime}} \\
& +\kappa\left(\nabla T_{k}, \nabla z\right)+\left(\mathbf{u}_{k} \cdot \nabla T_{k}, z\right) \\
& +\left(\mathbf{u}_{k} \cdot \nabla T^{\delta}, z\right)+\left(\mathbf{u}^{\varepsilon} \cdot \nabla T_{k}, z\right) \\
& +\kappa \int_{\Gamma_{5}} \gamma T_{k} \cdot z d \Gamma-\langle\widehat{g}, z\rangle_{H^{\prime}}
\end{aligned}
$$

for all $\mathbf{w}=(\mathbf{v}, z) \in \mathbb{X}_{k}$. By replacing $\mathbf{w}$ by $\left(\mathbf{u}_{k}, T_{k}\right)$ in (19) and using Lemma 3 , we obtain

$$
\begin{aligned}
\left(P\left(\mathbf{w}_{k}\right), \mathbf{w}_{k}\right)_{\mathbb{X}_{k}} \geq & 2 v\left\|\mathbf{u}_{k}\right\|_{\widetilde{\mathbf{H}}_{\sigma}^{1}}^{2}-\varepsilon\left\|\mathbf{u}_{k}\right\|_{\widetilde{\mathbf{H}}_{\sigma}^{1}}^{2}+\kappa\left\|T_{k}\right\|_{H_{0}}^{2} \\
& +\beta\left(\mathbf{G} T_{k}, \mathbf{u}_{k}\right)+\left(\mathbf{u}_{k} \cdot \nabla T^{\delta}, T_{k}\right) \\
& +\kappa \int_{\Gamma_{5}} \gamma T_{k} \cdot T_{k} d \Gamma-\left\langle\widehat{\mathbf{f}}, \mathbf{u}_{k}\right\rangle_{\widetilde{\mathbf{H}}_{\sigma}^{\prime}} \\
& -\left\langle\widehat{g}, T_{k}\right\rangle_{H^{\prime}} .
\end{aligned}
$$

Now, we will bind the right-hand side of (20). Since $\left\|\mathbf{u}_{k}\right\|_{\widetilde{\mathbf{H}}_{\sigma}^{1}}^{2}+$ $\left\|T_{k}\right\|_{H_{0}}^{2}=\left\|\mathbf{w}_{k}\right\|_{\mathbb{X}_{k}}^{2}$, then by using the Hölder and Young inequalities we have

$$
\begin{aligned}
& \left|\left\langle\widehat{\mathbf{f}}, \mathbf{u}_{k}\right\rangle_{\widetilde{\mathbf{H}}_{\sigma}^{\prime}}+\left\langle\hat{g}, T_{k}\right\rangle_{H^{\prime}}\right| \\
& \quad \leq\|\mathbf{f}\|_{\mathbf{H}_{\sigma}^{\prime}}\left\|\mathbf{u}_{k}\right\|_{\widetilde{\mathbf{H}}_{\sigma}^{1}}+\|\widehat{g}\|_{H^{\prime}}\left\|T_{k}\right\|_{H^{\prime}} \\
& \quad \leq\|(\widehat{\mathbf{f}}, \hat{g})\|_{\mathbb{X}^{\prime}}\left\|\mathbf{w}_{k}\right\|_{\mathbb{X}_{k}} .
\end{aligned}
$$

Applying the Hölder and Young inequalities, and taking into account the inequalities $\|\mathbf{u}\|_{4} \leq C\|\nabla \mathbf{u}\|,\|\nabla \mathbf{u}\| \leq C\|D(\mathbf{u})\|$, and $\left\|T^{\delta}\right\|_{4} \leq \delta$, we obtain

$$
\begin{aligned}
\left|\beta\left(\mathbf{G} T_{k}, \mathbf{u}_{k}\right)\right| & \leq \beta\|\mathbf{G}\|_{\infty}\left\|T_{k}\right\|\left\|\mathbf{u}_{k}\right\| \\
& \leq \beta C\|\mathbf{G}\|_{\infty}\left\|T_{k}\right\|_{H_{0}}\left\|\mathbf{u}_{k}\right\|_{\widetilde{\mathbf{H}}_{\sigma}^{1}} \\
& \leq \beta C\|\mathbf{G}\|_{\infty}\left(\left\|\mathbf{u}_{k}\right\|_{\widetilde{\mathbf{H}}_{\sigma}^{1}}^{2}+\left\|T_{k}\right\|_{H_{0}}^{2}\right), \\
\left|\kappa \int_{\Gamma_{5}} \gamma T_{k} \cdot T_{k} d \Gamma\right| & \leq \kappa\|\gamma\|_{L^{\infty}\left(\Gamma_{5}\right)}\left\|T_{k}\right\|_{L^{2}\left(\Gamma_{5}\right)}^{2} \\
& \leq \kappa C\|\gamma\|_{L^{\infty}\left(\Gamma_{5}\right)}\left\|T_{k}\right\|_{H_{0}}^{2}, \\
\left|\left(\mathbf{u}_{k} \cdot \nabla T^{\delta}, T_{k}\right)\right| & =\left|\left(\mathbf{u}_{k} \cdot \nabla T_{k}, T^{\delta}\right)\right| \\
& \leq\left\|\mathbf{u}_{k}\right\|_{4}\left\|\nabla T_{k}\right\|\left\|T^{\delta}\right\|_{4} \\
& \leq \delta C\left\|\mathbf{u}_{k}\right\|_{\widetilde{\mathbf{H}}_{\sigma}}\left\|T_{k}\right\|_{H_{0}} \\
& \leq \delta C\left(\left\|\mathbf{u}_{k}\right\|_{\widetilde{\mathbf{H}}_{\sigma}}^{2}+\left\|T_{k}\right\|_{H_{0}}^{2}\right) .
\end{aligned}
$$


By replacing (21) and (22) in (20) and taking into account (11), we have

$$
\begin{aligned}
\left(P\left(\mathbf{w}_{k}\right), \mathbf{w}_{k}\right) & )_{\mathbb{X}_{k}} \\
\geq & (2 \nu-\varepsilon)\left\|\mathbf{u}_{k}\right\|_{\widetilde{\mathbf{H}}_{\sigma}^{1}}^{2}+\left(\kappa-\kappa C\|\gamma\|_{L^{\infty}\left(\Gamma_{5}\right)}\right)\left\|T_{k}\right\|_{H_{0}}^{2} \\
& -\beta C\|\mathbf{G}\|_{\infty}\left(\left\|\mathbf{u}_{k}\right\|_{\widetilde{\mathbf{H}}_{\sigma}^{1}}^{2}+\left\|T_{k}\right\|_{H_{0}}^{2}\right) \\
& -\delta C\left(\left\|\mathbf{u}_{k}\right\|_{\widetilde{\mathbf{H}}_{\sigma}}^{2}+\left\|T_{k}\right\|_{H_{0}}^{2}\right)-\|(\widehat{\mathbf{f}}, \widehat{g})\|_{\mathbb{X}^{\prime}}\left\|\mathbf{w}_{k}\right\|_{\mathbb{X}_{k}} \\
\geq & \min \left\{2 v-\varepsilon, \kappa-\kappa C\|\gamma\|_{L^{\infty}\left(\Gamma_{5}\right)}\right\}\left\|\mathbf{w}_{k}\right\|_{\mathbb{X}_{k}}^{2} \\
& -\left(\beta C\|\mathbf{G}\|_{\infty}+\delta C\right)\left\|\mathbf{w}_{k}\right\|_{\mathbb{X}_{k}}^{2}-\|(\widehat{\mathbf{f}}, \widehat{g})\|_{\mathbb{X}^{\prime}}\left\|\mathbf{w}_{k}\right\|_{\mathbb{X}_{k}} \\
\geq & \left\|\mathbf{w}_{k}\right\|_{\mathbb{X}_{k}}\left[\widetilde{\delta}\left\|\mathbf{w}_{k}\right\|_{\mathbb{X}_{k}}-\|(\widehat{\mathbf{f}}, \widehat{g})\|_{\mathbb{X}^{\prime}}\right],
\end{aligned}
$$

where, by hypothesis,

$$
\begin{aligned}
\widetilde{\delta}:= & \min \left\{2 \nu-\varepsilon, \kappa-\kappa C\|\gamma\|_{L^{\infty}\left(\Gamma_{5}\right)}\right\} \\
& -\left(\beta C\|\mathbf{G}\|_{\infty}+\delta C\right)>0,
\end{aligned}
$$

for $\varepsilon$ small enough. Then, for $\left\|\mathbf{w}_{k}\right\|_{\mathbb{X}_{k}}=K$ with $K>$ $(1 / \widetilde{\delta})\|(\widehat{\mathbf{f}}, \widehat{g})\|_{\mathbb{X}^{\prime}}$, from (23) it follows that

$$
\left(P\left(\mathbf{w}_{k}\right), \mathbf{w}_{k}\right)_{\mathbb{X}_{k}} \geq K\left(\widetilde{\delta} K-\|(\widehat{\mathbf{f}}, \widehat{g})\|_{X^{\prime}}\right)>0 .
$$

Thus, by Lemma 4 , there exists $\left(\mathbf{u}_{k}, T_{k}\right) \in \mathbb{X}_{k}$ such that $\left\|\left(\mathbf{u}_{k}, T_{k}\right)\right\|_{\mathbb{X}_{k}} \geq K$ and

$$
\left(P\left(\mathbf{u}_{k}, T_{k}\right),(\mathbf{v}, z)\right)_{\mathbb{X}_{k}}=0 \quad \forall(\mathbf{v}, z) \in \mathbb{X}_{k} .
$$

Moreover, since $\left(\mathbf{v}_{i}, 0\right),\left(\mathbf{0}, z_{i}\right) \in \mathbb{X}_{k}$, from (19) and (26), we conclude that $\left(\mathbf{u}_{k}, T_{k}\right)$ is a solution to (18).

We now pass to the limit. Since $\mathbf{w}_{k}=\left(\mathbf{u}_{k}, T_{k}\right) \in \mathbb{X}_{k}$, then (26) implies $\left(P\left(\mathbf{w}_{k}\right), \mathbf{w}_{k}\right)_{\mathbb{X}_{k}}=0$ and, from (23), we conclude that

$$
\left\|\left(\mathbf{u}_{k}, T_{k}\right)\right\|_{\mathbb{X}_{k}} \leq \frac{1}{\widetilde{\delta}}\|(\widehat{\mathbf{f}}, \widehat{g})\|_{\mathbb{X}^{\prime}} .
$$

Thus, from (27), we conclude that the sequence $\left\{\left(\mathbf{u}_{k}, T_{k}\right)\right\}_{k \geq 1}$ is bounded in $\mathbb{X}$; then there exists a subsequence, still denoted by $\left\{\left(\mathbf{u}_{k}, T_{k}\right)\right\}_{k \geq 1}$, and some element $(\widehat{\mathbf{u}}, \widehat{T}) \in \mathbb{X}$, such that when $k \rightarrow \infty$,

$$
\begin{aligned}
& \mathbf{u}_{k} \longrightarrow \widehat{\mathbf{u}} \text { weakly in } \widetilde{\mathbf{H}}_{\sigma}^{1} \text { and strongly in } \mathbf{L}^{2}(\Omega), \\
& T_{k} \longrightarrow \widehat{T} \text { weakly in } H_{0} \text { and strongly in } L^{2}(\Omega) .
\end{aligned}
$$

The convergences (28) allow us to pass to the limit in (18) and to prove that $(\widehat{\mathbf{u}}, \widehat{T})$ is a solution to system (13). Moreover, from (27), we have

$$
\|(\widehat{\mathbf{u}}, \widehat{T})\|_{\mathbb{X}} \leq \liminf _{k \rightarrow \infty}\left\|\left(\mathbf{u}_{k}, T_{k}\right)\right\|_{\mathbb{X}_{k}} \leq \frac{1}{\widetilde{\delta}}\|(\widehat{\mathbf{f}}, \widehat{g})\|_{\mathbb{X}^{\prime}},
$$

which implies (16).
Theorem 6 (existence of weak solutions). Under the conditions of Theorem 5, there exists a solution $(\mathbf{u}, T) \in \mathbf{H}_{\sigma}^{1} \times H^{1}(\Omega)$ for system (7)-(9). Moreover, the following estimate holds:

$$
\|\mathbf{u}\|_{\mathbf{H}_{\sigma}^{1}}+\|T\|_{H^{1}} \leq \widetilde{C}_{1} \Theta
$$

where $\widetilde{C}_{1}$ is a positive constant which depends only on $\Omega, v, \kappa$, $\beta,\|\mathbf{G}\|_{\infty}$, and $\|\gamma\|_{L^{\infty}\left(\Gamma_{5}\right)}$ and $\Theta=\left\|\mathbf{u}_{\mathbf{g}_{1}}\right\|_{\mathbf{H}^{1 / 2}\left(\Gamma \backslash \Gamma_{3}\right)}+\left\|g_{2}\right\|_{H^{1 / 2}\left(\Gamma_{4}\right)}+$ $\left\|\mathbf{u}_{\mathbf{g}_{1}}\right\|_{\mathbf{H}^{1 / 2}\left(\Gamma \mid \Gamma_{3}\right)}^{2}+\left\|g_{2}\right\|_{H^{1 / 2}\left(\Gamma_{4}\right)}^{2}+\|\varphi\|_{L^{2}\left(\Gamma_{5}\right)}+\|(\mathbf{f}, g)\|_{\mathbb{X}^{\prime}}$.

Proof. As a consequence of Theorem 5, we have the fact that there exists a solution $(\mathbf{u}, T) \in \mathbf{H}_{\sigma}^{1} \times H^{1}(\Omega)$ for system (7)-(9), where $\mathbf{u}=\widehat{\mathbf{u}}+\mathbf{u}^{\varepsilon}$ and $T=\widehat{T}+T^{\delta}$, with $(\widehat{\mathbf{u}}, \widehat{T}) \in \mathbb{X}$ being a solution of (13) given in Theorem 5. Thus, from (16), we have

$$
\begin{aligned}
\|\mathbf{u}\|_{\mathbf{H}_{\sigma}^{1}}+\|T\|_{H^{1}} \leq & \|\widehat{\mathbf{u}}\|_{\widetilde{\mathbf{H}}_{\sigma}^{1}}+\|\widehat{T}\|_{H_{0}}+\left\|\mathbf{u}^{\varepsilon}\right\|_{\mathbf{H}_{\sigma}^{1}}+\left\|T^{\delta}\right\|_{H^{1}} \\
\leq & \sqrt{2}\|(\widehat{\mathbf{u}}, \widehat{T})\|_{\mathbb{X}^{\prime}}+\sqrt{2}\left\|\left(\mathbf{u}^{\varepsilon}, T^{\delta}\right)\right\|_{\mathbf{H}_{\sigma}^{1} \times H^{1}} \\
\leq & \frac{\sqrt{2}}{\delta}\|(\widehat{\mathbf{f}}, \widehat{g})\|_{\mathbb{X}^{\prime}} \\
& +\sqrt{2}\left\|\left(\mathbf{u}^{\varepsilon}, T^{\delta}\right)\right\|_{\mathbf{H}_{\sigma}^{1} \times H^{1}} .
\end{aligned}
$$

From (14) we can obtain

$$
\begin{aligned}
& \|(\widehat{\mathbf{f}}, \widehat{g})\|_{\mathbb{X}^{\prime}} \leq C_{1}\left(\left\|\left(\mathbf{u}^{\varepsilon}, T^{\delta}\right)\right\|_{\mathbf{H}_{\sigma}^{1} \times H^{1}}+\left\|\left(\mathbf{u}^{\varepsilon}, T^{\delta}\right)\right\|_{\mathbf{H}_{\sigma}^{1} \times H^{1}}^{2}\right. \\
& \left.\quad+\|\varphi\|_{L^{2}\left(\Gamma_{5}\right)}+\|(\mathbf{f}, g)\|_{\mathbb{X}^{\prime}}\right),
\end{aligned}
$$

where $C_{1}$ is a positive constant which depends on $\beta,\|\mathbf{G}\|_{\infty},\|\gamma\|_{L^{\infty}\left(\Gamma_{5}\right)}, v$, and $\kappa$. Thus, from (31), we have

$$
\begin{aligned}
\|\mathbf{u}\|_{\mathbf{H}_{\sigma}^{1}} & +\|T\|_{H^{1}} \\
\leq & C_{1} \frac{C}{\delta}\left(\left\|\left(\mathbf{u}^{\varepsilon}, T^{\delta}\right)\right\|_{\mathbf{H}_{\sigma}^{1} \times H^{1}}+\left\|\left(\mathbf{u}^{\varepsilon}, T^{\delta}\right)\right\|_{\mathbf{H}_{\sigma}^{1} \times H^{1}}^{2}\right) \\
& +C_{1} \frac{C}{\delta}\left(\|\varphi\|_{L^{2}\left(\Gamma_{5}\right)}+\|(\mathbf{f}, g)\|_{\mathbb{X}^{\prime}}\right) .
\end{aligned}
$$

Since $\left\|\left(\mathbf{u}^{\varepsilon}, T^{\delta}\right)\right\|_{\mathbf{H}_{\sigma}^{1} \times H^{1}} \leq\left\|\mathbf{u}^{\varepsilon}\right\|_{\mathbf{H}_{\sigma}^{1}}+\left\|T^{\delta}\right\|_{H^{1}}$, then, by Lemma 3, we have

$$
\begin{aligned}
& \left\|\left(\mathbf{u}^{\varepsilon}, T^{\delta}\right)\right\|_{\mathbf{H}_{\sigma}^{1} \times H^{1}} \leq C\left(\left\|\mathbf{u}_{\mathbf{g}_{1}}\right\|_{\mathbf{H}^{1 / 2}\left(\Gamma \backslash \Gamma_{3}\right)}+\left\|g_{2}\right\|_{H^{1 / 2}\left(\Gamma_{4}\right)}\right), \\
& \left\|\left(\mathbf{u}^{\varepsilon}, T^{\delta}\right)\right\|_{\mathbf{H}_{\sigma}^{1} \times H^{1}}^{2} \leq C\left(\left\|\mathbf{u}_{\mathbf{g}_{1}}\right\|_{\mathbf{H}^{1 / 2}\left(\Gamma \backslash \Gamma_{3}\right)}^{2}+\left\|g_{2}\right\|_{H^{1 / 2}\left(\Gamma_{4}\right)}^{2}\right) .
\end{aligned}
$$

Therefore, the above inequalities together with (33) imply (30).

Remark 7. Since $\operatorname{Re}=1 / v$, then condition (15) shows us that the existence of solution for the system (7)-(9) is guaranteed when the Reynolds number is small (the flow is laminar). 
Theorem 8 (uniqueness of solutions). Under conditions of Theorem 6 , if $\nu, \kappa$ are large enough and $\|\gamma\|_{L^{\infty}\left(\Gamma_{5}\right)}$ is small enough such that

$$
\begin{aligned}
& \min \left\{2 \nu-\beta C\|\mathbf{G}\|_{\infty}, \kappa-\beta C\|\mathbf{G}\|_{\infty}-\kappa C\|\gamma\|_{L^{\infty}\left(\Gamma_{5}\right)}\right\} \\
& >C \widetilde{C}_{1} \Theta
\end{aligned}
$$

where $C$ is a positive constant that depends on the domain $\Omega$ and $\widetilde{C}_{1}, \Theta$ are given in (30), then the solution $(\mathbf{u}, T) \in \mathbf{H}_{\sigma}^{1} \times$ $H^{1}(\Omega)$ of system (7)-(9) provided by Theorem 6 is unique.

Proof. Let $\left(\mathbf{u}_{1}, T_{2}\right),\left(\mathbf{u}_{2}, T_{2}\right) \in \mathbf{H}_{\sigma}^{1} \times H^{1}(\Omega)$ be two solutions of system (7)-(9). Then, subtracting the corresponding equations and denoting $\mathbf{u}=\mathbf{u}_{1}-\mathbf{u}_{2}$ and $T=T_{1}-T_{2}$, we obtain $(\mathbf{u}, T) \in \mathbb{X}$ and the following system:

$$
\begin{aligned}
& 2 \nu(D(\mathbf{u}), D(\mathbf{v}))+2 \alpha \nu \int_{\Gamma_{3}} \mathbf{u} \cdot \mathbf{v} d \Gamma+\left(\mathbf{u}_{1} \cdot \nabla \mathbf{u}, \mathbf{v}\right) \\
& +\left(\mathbf{u} \cdot \nabla \mathbf{u}_{2}, \mathbf{v}\right)+\beta(\mathbf{G} T, \mathbf{v})=0 \quad \forall \mathbf{v} \in \widetilde{\mathbf{H}}_{\sigma}^{1}, \\
& \kappa(\nabla T, \nabla z)+\left(\mathbf{u}_{1} \cdot \nabla T, z\right)+\left(\mathbf{u} \cdot \nabla T_{2}, z\right) \\
& \quad+\kappa \int_{\Gamma_{5}} \gamma T \cdot z d \Gamma=0 \quad \forall z \in H_{0} .
\end{aligned}
$$

Now, by replacing $\mathbf{v}=\mathbf{u}$ in (36) and $z=T$ in (37) and adding the results, we have

$$
\begin{aligned}
2 \nu\|\mathbf{u}\|_{\widetilde{\mathbf{H}}_{\sigma}^{1}}^{2}+\kappa\|T\|_{H_{0}}^{2} \leq & \left|\left(\mathbf{u} \cdot \nabla \mathbf{u}_{2}, \mathbf{u}\right)\right|+\beta|(\mathbf{G T}, \mathbf{u})| \\
& +\left|\left(\mathbf{u} \cdot \nabla T_{2}, T\right)\right| \\
& +\kappa\left|\int_{\Gamma_{5}} \gamma T \cdot T d \Gamma\right| .
\end{aligned}
$$

By using the Hölder and Young inequalities, we obtain

$$
\begin{aligned}
& \left|\left(\mathbf{u} \cdot \nabla \mathbf{u}_{2}, \mathbf{u}\right)\right| \leq\|\mathbf{u}\|_{3}\left\|\nabla \mathbf{u}_{2}\right\|\|\mathbf{u}\|_{6} \\
& \leq C\|\mathbf{u}\|_{\widetilde{\mathbf{H}}_{\sigma}^{1}}^{2}\left\|\mathbf{u}_{2}\right\|_{\mathbf{H}_{\sigma}^{1}}, \\
& \beta|(\mathbf{G} T, \mathbf{u})| \leq \beta\|\mathbf{G}\|_{\infty}\|T\|\|\mathbf{u}\| \\
& \leq \beta C\|\mathbf{G}\|_{\infty}\|T\|_{H_{0}}\|\mathbf{u}\|_{\widetilde{\mathbf{H}}_{\sigma}^{1}} \\
& \leq \beta C\|\mathbf{G}\|_{\infty}\left(\|\mathbf{u}\|_{\widetilde{\mathbf{H}}_{\sigma}^{1}}^{2}+\|T\|_{H_{0}}^{2}\right), \\
& \left|\left(\mathbf{u} \cdot \nabla T_{2}, T\right)\right| \leq\|\mathbf{u}\|_{3}\left\|\nabla T_{2}\right\|\|T\|_{6} \\
& \leq C\|\mathbf{u}\|_{\widetilde{\mathbf{H}}_{\sigma}^{1}}\left\|T_{2}\right\|_{H^{1}}\|T\|_{H_{0}} \\
& \leq C\left\|T_{2}\right\|_{H^{1}}\left(\|\mathbf{u}\|_{\widetilde{\mathbf{H}}_{\sigma}^{1}}^{2}+\|T\|_{H_{0}}^{2}\right), \\
& \kappa\left|\int_{\Gamma_{5}} \gamma T \cdot T d \Gamma\right| \leq \kappa\|\gamma\|_{L^{\infty}\left(\Gamma_{5}\right)}\|T\|_{L^{2}\left(\Gamma_{5}\right)}^{2} \\
& \leq \kappa C\|\gamma\|_{L^{\infty}\left(\Gamma_{5}\right)}\|T\|_{H_{0}}^{2} .
\end{aligned}
$$

By replacing (39) in (38), we can obtain

$$
\begin{aligned}
(2 \nu & \left.-\beta C\|\mathbf{G}\|_{\infty}\right)\|\mathbf{u}\|_{\widetilde{\mathbf{H}}_{\sigma}^{1}}^{2} \\
& +\left(\kappa-\beta C\|\mathbf{G}\|_{\infty}-\kappa C\|\gamma\|_{L^{\infty}\left(\Gamma_{5}\right)}\right)\|T\|_{H_{0}}^{2} \\
\leq & C\left(\left\|\mathbf{u}_{2}\right\|_{\mathbf{H}_{\sigma}^{1}}+\left\|T_{2}\right\|_{H^{1}}\right)\|\mathbf{u}\|_{\widetilde{\mathbf{H}}_{\sigma}^{1}}^{2}+C\left\|T_{2}\right\|_{H^{1}}\|T\|_{H_{0}}^{2} .
\end{aligned}
$$

From (30) we have $\left\|\mathbf{u}_{2}\right\|_{\mathbf{H}_{\sigma}^{1}}+\left\|T_{2}\right\|_{H^{1}} \leq \widetilde{C}_{1} \Theta$; then (40) implies

$$
\begin{aligned}
(2 \nu & \left.-\beta C\|\mathbf{G}\|_{\infty}\right)\|\mathbf{u}\|_{\widetilde{\mathbf{H}}_{\sigma}^{1}}^{2} \\
& +\left(\kappa-\beta C\|\mathbf{G}\|_{\infty}-\kappa C\|\gamma\|_{L^{\infty}\left(\Gamma_{5}\right)}\right)\|T\|_{H_{0}}^{2} \\
\leq & C \widetilde{C}_{1} \Theta\left(\|\mathbf{u}\|_{\widetilde{\mathbf{H}}_{\sigma}^{1}}^{2}+\|T\|_{H_{0}}^{2}\right),
\end{aligned}
$$

and thus

$$
\begin{aligned}
(2 \nu & \left.-\beta C\|\mathbf{G}\|_{\infty}-C \widetilde{C}_{1} \Theta\right)\|\mathbf{u}\|_{\widetilde{\mathbf{H}}_{\sigma}^{1}}^{2} \\
& +\left(\kappa-\beta C\|\mathbf{G}\|_{\infty}-\kappa C\|\gamma\|_{L^{\infty}\left(\Gamma_{5}\right)}-C \widetilde{C}_{1} \Theta\right)\|T\|_{H_{0}}^{2}
\end{aligned}
$$

$\leq 0$.

Therefore, if $\min \left\{2 \nu-\beta C\|\mathbf{G}\|_{\infty}, \kappa-\beta C\|\mathbf{G}\|_{\infty}-\kappa C\|\gamma\|_{L^{\infty}\left(\Gamma_{5}\right)}\right\}>$ $C \widetilde{C_{1}} \Theta$, from (42), we have $\|\mathbf{u}\|_{\widetilde{\mathbf{H}}_{\sigma}^{1}}=\|T\|_{H_{0}}=0$, that is, $\mathbf{u}_{1}=\mathbf{u}_{2}$ and $T_{1}=T_{2}$, and the theorem is proved.

Remark 9. The condition (35) assures us of the uniqueness of solution of system (7)-(9) when the viscosity $v$ and the temperature conduction coefficient $\kappa$ are large enough, which implies that the Rayleigh number is small. That is, the uniqueness of weak solution is obtained when the heat transfer is produced primarily by conduction.

\section{The Optimal Control Problem}

In this section the statement of the boundary control problem to study is established. We suppose that $\mathcal{U}_{1} \subset \widetilde{\mathbf{H}}_{00}^{1 / 2}\left(\Gamma_{2}\right)$ and $\mathcal{U}_{2} \subset H_{00}^{1 / 2}\left(\Gamma_{4}\right)$ are nonempty sets. We consider data $(\mathbf{f}, g) \in$ $\mathbb{X}^{\prime}, \mathbf{G} \in \mathbf{L}^{\infty}(\Omega), \mathbf{u}_{0} \in \widetilde{\mathbf{H}}_{00}^{1 / 2}\left(\Gamma_{1}\right), \varphi \in L^{2}\left(\Gamma_{5}\right), \gamma \in L^{\infty}\left(\Gamma_{5}\right)$, and the functions $\mathbf{g}_{1} \in \mathcal{U}_{1}, g_{2} \in \mathcal{U}_{2}$, describing the Dirichlet boundary controls for $\mathbf{u}$ on $\Gamma_{2}$ and $T$ on $\Gamma_{4}$, respectively. For simplicity, here and hereinbelow, we will use the product space $\mathbb{H}=\mathbf{H}_{\sigma}^{1} \times H^{1}(\Omega)$, and we consider the following objective functional $J: \mathbb{U} \times \mathcal{U}_{1} \times \mathcal{U}_{2} \rightarrow \mathbb{R}$ defined by

$$
\begin{aligned}
J\left(\mathbf{u}, T, \mathbf{g}_{1}, g_{2}\right)= & \widehat{J}(\mathbf{u}, T)+\frac{b_{1}}{2}\left\|\mathbf{g}_{1}\right\|_{\mathbf{H}^{1 / 2}\left(\Gamma_{2}\right)}^{2} \\
& +\frac{b_{2}}{2}\left\|g_{2}\right\|_{H^{1 / 2}\left(\Gamma_{4}\right)}^{2},
\end{aligned}
$$

where $\widehat{J}: \mathbf{H}_{\sigma}^{1} \times H^{1}(\Omega) \rightarrow \mathbb{R}$ is a weakly lower semicontinuous functional, and the nonnegative constants $b_{1}, b_{2}$ measure the cost of the controls. 
The following are examples of the weakly lower semicontinuous functional in (43) most interesting from a physical viewpoint:

$$
\begin{aligned}
& J_{1}(\mathbf{u})=\frac{1}{2} \int_{\Omega}\left|\mathbf{u}-\mathbf{u}_{d}\right|^{2} d \Omega, \\
& J_{2}(T)=\frac{1}{2} \int_{\Omega}\left|T-T_{d}\right|^{2} d \Omega, \\
& J_{3}(\mathbf{u})=\frac{v}{2} \int_{\Omega}\left|\nabla \mathbf{u}+\nabla^{T} \mathbf{u}\right|^{2} d \Omega,
\end{aligned}
$$

Find $\quad\left(\mathbf{u}, T, \mathbf{g}_{1}, g_{2}\right) \in \mathbb{H}$ where $\mathbf{u}_{d} \in \mathbf{L}^{2}(\Omega)$ and $T_{d} \in L^{2}(\Omega)$ are the desired states. The functionals $J_{1}$ and $J_{2}$ describe, respectively, the deviation of the velocity of flow from a given velocity and the deviation of the temperature from a given temperature. The functional $J_{3}$ describes the resistance in a fluid due to viscous friction (see [22]).

For simplicity we will do the study with $\widehat{J}=J_{1}+J_{2}$; also the study can be done considering $\widehat{J}$ as the sum of two or more functionals given in (44). Thus, we define the following constrained minimization problem related to system (7)-(9):

such that $J\left(\mathbf{u}, T, \mathbf{g}_{1}, g_{2}\right)=\frac{1}{2}\left\|\mathbf{u}-\mathbf{u}_{d}\right\|^{2}+\frac{1}{2}\left\|T-T_{d}\right\|^{2}+\frac{b_{1}}{2}\left\|\mathbf{g}_{1}\right\|_{\mathbf{H}^{1 / 2}\left(\Gamma_{2}\right)}^{2}+\frac{b_{2}}{2}\left\|g_{2}\right\|_{H^{1 / 2}\left(\Gamma_{4}\right)}^{2}$

reaches its minimum over the weak solutions of system (1)-(4).

The set of admissible solutions of optimal control problem (45) is defined by

$$
\begin{aligned}
\mathcal{S}_{\mathrm{ad}} & =\left\{\mathbf{s}=\left(\mathbf{u}, T, \mathbf{g}_{1}, g_{2}\right) \in \mathbb{H} \times \mathcal{U}_{\mathrm{ad}} \text { such that } J(\mathbf{s})\right. \\
<\infty & \text { and s satisfies }(7)-(9)\},
\end{aligned}
$$

where $\mathcal{U}_{\mathrm{ad}}:=\mathcal{U}_{1} \times \mathcal{U}_{2}$. A similar control problem has been studied in $[20,21]$ for micropolar fluids. In [20] a threedimensional domain was considered with density constant; while in [21] the problem was studied in a two-dimensional domain, where the fluid density was considered as an extra variable in the system.

4.1. Existence of Optimal Solution. In this subsection we will prove the existence of an optimal solution for problem (45). For this purpose we introduce the following result. The proof follows a technique that is standard for optimal control problems. We will repeat it for convenience of the reader.

Theorem 10. Under the hypotheses of Theorem 6, and also assuming that the conditions are satisfied:

(i) $b_{i} \geq 0$, for $i=1,2$,

$$
\begin{aligned}
& \mathcal{U}_{1} \subset \widetilde{\mathbf{H}}_{00}^{1 / 2}\left(\Gamma_{2}\right), \\
& \mathscr{U}_{2} \subset H_{00}^{1 / 2}\left(\Gamma_{4}\right) \\
& \quad \text { are bounded, closed and convex set; }
\end{aligned}
$$

(ii) $b_{i}>0$, for $i=1,2$,

$$
\begin{aligned}
& \mathcal{U}_{1} \subset \widetilde{\mathbf{H}}_{00}^{1 / 2}\left(\Gamma_{2}\right), \\
& \mathcal{U}_{2} \subset H_{00}^{1 / 2}\left(\Gamma_{4}\right)
\end{aligned}
$$

are closed and convex set; then problem (45) has at least one optimal solution; that is, there exist $\widetilde{\mathbf{s}}=\left(\widetilde{\mathbf{u}}, \widetilde{T}, \widetilde{\mathbf{g}}_{1}, \widetilde{g}_{2}\right) \in \mathcal{S}_{\text {ad }}$ such that

$$
J\left(\widetilde{\mathbf{u}}, \widetilde{T}, \widetilde{\mathbf{g}}_{1}, \widetilde{g}_{2}\right)=\min _{\mathbf{s} \in \mathcal{S}_{a d}} J\left(\mathbf{u}, T, \mathbf{g}_{1}, g_{2}\right) .
$$

Proof. From Theorem 6 we have that the set of admissible solutions $S_{\mathrm{ad}} \neq \emptyset$, and since the functional $J$ is bounded below, there exists a minimizing sequence $\left\{\mathbf{s}^{m}=\left(\mathbf{u}^{m}, T^{m}, \mathbf{g}_{1}^{m}, g_{2}^{m}\right)\right\}_{m>1} \in \mathcal{S}_{\text {ad }}, m \in \mathbb{N}$, such that $\lim _{m \rightarrow \infty} J\left(\mathbf{s}^{m}\right)=\inf _{\mathbf{s} \in \mathcal{S}_{\mathrm{ad}}} J(\mathbf{s})$. Also, by definition of set $\mathcal{S}_{\mathrm{ad}}$, we have the fact that $\mathbf{s}^{m}$ satisfies system (7)-(9); that is,

$$
\begin{aligned}
& 2 \nu\left(D\left(\mathbf{u}^{m}\right), D(\mathbf{v})\right)+2 \alpha \nu \int_{\Gamma_{3}} \mathbf{u}^{m} \cdot \mathbf{v} d \Gamma \\
& \quad+\left(\mathbf{u}^{m} \cdot \nabla \mathbf{u}^{m}, \mathbf{v}\right)+\beta\left(\mathbf{G} T^{m}, \mathbf{v}\right)=\langle\mathbf{f}, \mathbf{v}\rangle_{\widetilde{\mathbf{H}}_{\sigma}^{\prime}}, \\
& \kappa\left(\nabla T^{m}, \nabla z\right)+\left(\mathbf{u}^{m} \cdot \nabla T^{m}, z\right) \\
& \quad+\kappa \int_{\Gamma_{5}} \gamma T^{m} \cdot z d \Gamma=\langle g, z\rangle_{H^{\prime}}+\langle\varphi, z\rangle_{\Gamma_{5}}, \\
& \mathbf{u}^{m}=\mathbf{u}_{\mathbf{g}_{1}^{m}} \text { on } \Gamma \backslash \Gamma_{3}, \\
& T^{m}=g_{2}^{m} \text { on } \Gamma_{4},
\end{aligned}
$$

for all $(\mathbf{v}, z) \in \mathbb{X}$. Now, if one of the conditions $(i)$ or (ii) in (47) is satisfied, then there exists a constant $C$, independent of $m$, such that $\left\|\mathbf{g}_{1}^{m}\right\|_{\mathbf{H}^{1 / 2}\left(\Gamma_{2}\right)}^{2}+\left\|g_{2}^{m}\right\|_{H^{1 / 2}\left(\Gamma_{4}\right)}^{2} \leq C$; thus, from (30), we conclude that $\left\|\mathbf{u}^{m}\right\|_{\mathbf{H}_{\sigma}^{1}}+\left\|T^{m}\right\|_{H^{1}} \leq C$. Therefore, since $\mathcal{U}_{\mathrm{ad}}$ is a closed convex subset of $\widetilde{\mathbf{H}}_{00}^{1 / 2}\left(\Gamma_{2}\right) \times H_{00}^{1 / 2}\left(\Gamma_{4}\right)$, there exists 
$\widetilde{\mathbf{s}}=\left(\widetilde{\mathbf{u}}, \widetilde{T}, \widetilde{\mathbf{g}}_{1}, \widetilde{g}_{2}\right) \in \mathbb{H} \times \mathcal{U}_{\text {ad }}$ such that, for some subsequence of $\left\{\mathbf{s}^{m}\right\}_{m \geq 1}$, still denoted by $\left\{\mathbf{s}^{m}\right\}_{m \geq 1}$, when $m \rightarrow \infty$, we have

$$
\begin{aligned}
& \mathbf{u}^{m} \longrightarrow \widetilde{\mathbf{u}} \text { weakly in } \mathbf{H}_{\sigma}^{1}, \\
& T^{m} \longrightarrow \widetilde{T} \text { weakly in } H^{1}(\Omega), \\
& \mathbf{g}_{1}^{m} \longrightarrow \widetilde{\mathbf{g}}_{1} \text { weakly in } \mathcal{U}_{1}, \\
& g_{2}^{m} \longrightarrow \widetilde{g}_{2} \text { weakly in } \mathcal{U}_{2} ;
\end{aligned}
$$

by standard Sobolev embeddings, we obtain that

$$
\begin{aligned}
& \mathbf{u}^{m} \longrightarrow \widetilde{\mathbf{u}} \text { strongly in } \mathbf{L}^{2}(\Omega), \\
& T^{m} \longrightarrow \widetilde{T} \text { strongly in } L^{2}(\Omega), \\
& \mathbf{g}_{1}^{m} \longrightarrow \widetilde{\mathbf{g}}_{1} \text { strongly in } \mathbf{L}^{2}\left(\Gamma_{2}\right), \\
& g_{2}^{m} \longrightarrow \widetilde{g}_{2} \text { strongly in } L^{2}\left(\Gamma_{4}\right) .
\end{aligned}
$$

Moreover, since $\mathbf{u}^{m}=\mathbf{u}_{0}$ on $\Gamma_{1}, \mathbf{u}^{m}=\mathbf{g}_{1}^{m}$ on $\Gamma_{2}$, and $T^{m}=$ $g_{2}^{m}$ on $\Gamma_{4}$, then from (51) it follows that $\widetilde{\mathbf{u}}=\mathbf{u}_{\widetilde{\mathbf{g}}_{1}}$ on $\Gamma \backslash \Gamma_{3}$ and $\widetilde{T}=\widetilde{g}_{2}$ on $\Gamma_{4}$; thus, $\widetilde{\mathbf{s}}$ satisfies the boundary conditions given in (9). A standard procedure permits passing the limit in (51), as $m$ goes to $\infty$, and proving that $\widetilde{\mathbf{s}}$ is solution of system (7)-(9). Consequently we have $\widetilde{\mathbf{s}} \in \mathcal{S}_{\text {ad }}$ and

$$
\lim _{m \rightarrow \infty} J\left(\mathbf{s}^{m}\right)=\inf _{\mathbf{s} \in S_{\mathrm{ad}}} J(\mathbf{s}) \leq J(\widetilde{\mathbf{s}}) .
$$

Also, since the functional $J$ is weakly lower semicontinuous on $\mathcal{S}_{\text {ad }}$, we have that $J(\widetilde{\mathbf{s}}) \leq \lim _{m \rightarrow \infty} \inf J\left(\mathbf{s}^{m}\right)$. Therefore, from (52) and last inequality we conclude $J\left(\widetilde{\mathbf{u}}, \widetilde{T}, \widetilde{\mathbf{g}}_{1}, \widetilde{g}_{2}\right)=$ $J(\widetilde{\mathbf{s}})=\min _{\mathbf{s} \in S_{\mathrm{ad}}} J(\mathbf{s})$, which implies the existence of an optimal solution to the control problem (45).

4.2. First-Order Necessary Optimality Conditions and an Optimality System. In order to obtain first-order optimality conditions, and thus derive an optimality system, we start by considering the following Banach space:

$$
\mathbb{Y}:=\mathbb{X}^{\prime} \times \widetilde{\mathbf{H}}_{00}^{1 / 2}\left(\Gamma \backslash \Gamma_{3}\right) \times H_{00}^{1 / 2}\left(\Gamma_{4}\right)
$$

and the vector operator $\mathbb{F}=\left(\mathbf{F}_{1}, F_{2}, \mathbf{F}_{3}, F_{4}\right): \mathbb{W} \times \mathcal{U}_{\text {ad }} \rightarrow \mathbb{Y}$, where $\mathbf{F}_{1}: \mathbb{H} \times \mathcal{U}_{\mathrm{ad}} \rightarrow \widetilde{\mathbf{H}}_{\sigma}^{\prime}, F_{2}: \mathbb{H} \times \mathscr{U}_{\mathrm{ad}} \rightarrow H^{\prime}, \mathbf{F}_{3}:$ $\mathbb{H} \times \mathcal{U}_{\mathrm{ad}} \rightarrow \widetilde{\mathbf{H}}_{00}^{1 / 2}\left(\Gamma \backslash \Gamma_{3}\right)$, and $F_{4}: \mathbb{H} \times \mathcal{U}_{\mathrm{ad}} \rightarrow H_{00}^{1 / 2}\left(\Gamma_{4}\right)$, at each point $\mathbf{s}=\left(\mathbf{u}, T, \mathbf{g}_{1}, g_{2}\right) \in \mathbb{H} \times \mathscr{U}_{\mathrm{ad}}$, are defined by

$$
\begin{aligned}
\left\langle\mathbf{F}_{1}(\mathbf{s}), \mathbf{v}\right\rangle_{\widetilde{\mathbf{H}}_{\sigma}^{\prime}}:= & 2 \nu(D(\mathbf{u}), D(\mathbf{v}))+2 \alpha \nu \int_{\Gamma_{3}} \mathbf{u} \cdot \mathbf{v} d \Gamma \\
& +(\mathbf{u} \cdot \nabla \mathbf{u}, \mathbf{v})+\beta(\mathbf{G} T, \mathbf{v}) \\
& -\langle\mathbf{f}, \mathbf{v}\rangle_{\widetilde{\mathbf{H}}_{\sigma}^{\prime}},
\end{aligned}
$$

$$
\begin{aligned}
\left\langle F_{2}(\mathbf{s}), z\right\rangle_{H^{\prime}}:= & \kappa(\nabla T, \nabla z)+(\mathbf{u} \cdot \nabla T, z) \\
& +\kappa \int_{\Gamma_{5}} \gamma T \cdot z d \Gamma \\
& -\left(\langle g, z\rangle_{H^{\prime}}+\langle\varphi, z\rangle_{\Gamma_{5}}\right), \\
\mathbf{F}_{3}(\mathbf{s}):= & \mathbf{u}_{|\Gamma| \Gamma_{3}}-\mathbf{u}_{\mathbf{g}_{1}}, \\
F_{4}(\mathbf{s}):= & T_{\mid \Gamma_{4}}-g_{2},
\end{aligned}
$$

for all $(\mathbf{v}, z) \in \mathbb{X}$. Thus, the optimal control problem (45) is rewritten as follows:

$$
\begin{aligned}
& \text { Find } \mathbf{s}=\left((\mathbf{u}, T),\left(\mathbf{g}_{1}, g_{2}\right)\right) \\
& \in \mathbb{H} \times \mathscr{U}_{\mathrm{ad}} \\
& \text { such that the functional } J\left(\mathbf{u}, T, \mathbf{g}_{1}, g_{2}\right) \\
& =\frac{1}{2}\left\|\mathbf{u}-\mathbf{u}_{d}\right\|^{2} \\
& +\frac{1}{2}\left\|T-T_{d}\right\|^{2} \\
& +\frac{b_{1}}{2}\left\|\mathbf{g}_{1}\right\|_{\mathbf{H}^{1 / 2}\left(\Gamma_{2}\right)}^{2} \\
& +\frac{b_{2}}{2}\left\|g_{2}\right\|_{H^{1 / 2}\left(\Gamma_{4}\right)}^{2} \\
& \text { that is, }\left\langle\mathbf{F}_{1}(\mathbf{s}), \mathbf{v}\right\rangle_{\widetilde{\mathbf{H}}_{\sigma}^{\prime}}=0 \text {, } \\
& \left\langle\mathbf{F}_{2}(\mathbf{s}), z\right\rangle_{H^{\prime}}=0 \text {, } \\
& \mathbf{F}_{3}(\mathbf{s})=\mathbf{0} \text {, } \\
& \mathbf{F}_{4}(\mathbf{s})=0 \text {, }
\end{aligned}
$$

for all $(\mathbf{v}, z) \in \mathbb{X}$.

Recall that problem (45) is a problem with constraint; one method to obtain necessary conditions of optimality is Lagrange method, which consists of transforming the problem (45) into an unrestricted one, using an auxiliary function (Lagrange functional or Lagrangian) and additional variables, called Lagrange multipliers. The Lagrangian associated with optimal control problem (45) is given by

$$
\begin{aligned}
\mathscr{L}(\mathbf{s}, \phi)= & J(\mathbf{s})-\left\langle\mathbf{F}_{1}(\mathbf{s}), \lambda\right\rangle_{\widetilde{\mathbf{H}}_{\sigma}^{\prime}}-\left\langle F_{2}(\mathbf{s}), \eta\right\rangle_{H^{\prime}} \\
& -\left\langle\chi, \mathbf{F}_{3}(\mathbf{s})\right\rangle_{\Gamma \backslash \Gamma_{3}}-\left\langle\vartheta, F_{4}(\mathbf{s})\right\rangle_{\Gamma_{4}},
\end{aligned}
$$

where $\phi=((\boldsymbol{\lambda}, \eta), \boldsymbol{\chi}, \vartheta) \in \mathbb{X} \times \widetilde{\mathbf{H}}_{00}^{-1 / 2}\left(\Gamma \backslash \Gamma_{3}\right) \times H_{00}^{-1 / 2}\left(\Gamma_{4}\right)$.

Concerning differentiation of the objective functional $J$ and the constraint operator $\mathbb{F}$, we have the following results.

Lemma 11. The functional $J$ is Fréchet differentiable with respect to the point $\mathbf{s}=\left((\mathbf{u}, T),\left(\mathbf{g}_{1}, g_{2}\right)\right) \in \mathbb{U} \times \mathcal{U}_{\text {ad }}$. Moreover, at an arbitrary point $\widetilde{\mathbf{s}}=\left((\widetilde{\mathbf{u}}, \widetilde{T}),\left(\widetilde{\mathbf{g}}_{1}, \widetilde{g}_{2}\right)\right) \in \mathbb{U} \times \mathcal{U}_{\text {ad }}$, the 
Fréchet derivative of $J$ with respect to $\mathbf{s}$ is a linear and bounded functional $J_{\mathbf{s}}(\widetilde{\mathbf{s}}): \mathbb{H} \times \mathscr{U}_{\text {ad }} \rightarrow \mathbb{R}$ such that, at each point $\mathbf{t}=((\boldsymbol{\omega}, \psi),(\boldsymbol{\varrho}, \tau)) \in \mathbb{W} \times \mathscr{U}_{a d}$, it is defined by

$$
\begin{aligned}
J_{\mathbf{s}}(\widetilde{\mathbf{s}}) \mathbf{t}= & \left(\widetilde{\mathbf{u}}-\mathbf{u}_{d}, \boldsymbol{\omega}\right)+\left(\widetilde{T}-T_{d}, \psi\right)+b_{1} \int_{\Gamma_{2}} \widetilde{\mathbf{g}}_{1} \cdot \varrho d \Gamma \\
& +b_{2} \int_{\Gamma_{4}} \widetilde{g}_{2} \cdot \tau d \Gamma .
\end{aligned}
$$

Lemma 12. The operator $\mathbb{F}$ is Fréchet differentiable with respect to the point $\mathbf{s}=\left((\mathbf{u}, T),\left(\mathbf{g}_{1}, g_{2}\right)\right) \in \mathbb{M} \times \mathcal{U}_{\text {ad }}$. Moreover, at an arbitrary point $\widetilde{\mathbf{s}}=\left((\widetilde{\mathbf{u}}, \widetilde{T}),\left(\widetilde{\mathbf{g}}_{1}, \widetilde{g}_{2}\right)\right) \in \mathbb{M} \times \mathcal{U}_{\text {ad }}$, the Fréchet derivative of $\mathbb{F}$ with respect to $\mathbf{s}$ is a linear and bounded operator $\mathbb{F}_{\mathbf{s}}(\widetilde{\mathbf{s}}): \mathbb{H} \times \mathcal{U}_{\text {ad }} \rightarrow \mathbb{R}$ such that, at each point $\mathbf{t}=((\boldsymbol{\omega}, \psi),(\varrho, \tau)) \in \mathbb{W} \times \mathscr{U}_{a d}$, it is defined by

$$
\begin{aligned}
\left\langle\mathbf{F}_{1 \mathbf{s}}(\widetilde{\mathbf{s}}) \mathbf{t}, \mathbf{v}\right\rangle_{\widetilde{\mathbf{H}}_{\sigma}^{\prime}=} & 2 \nu(D(\boldsymbol{\omega}), D(\mathbf{v}))+2 \alpha \nu \int_{\Gamma_{3}} \boldsymbol{\omega} \cdot \mathbf{v} d \Gamma \\
& +(\widetilde{\mathbf{u}} \cdot \nabla \boldsymbol{\omega}, \mathbf{v})+(\boldsymbol{\omega} \cdot \nabla \widetilde{\mathbf{u}}, \mathbf{v}) \\
& +\beta(\mathbf{G} \psi, \mathbf{v}), \\
\left\langle F_{2 \mathbf{s}}(\widetilde{\mathbf{s}}) \mathbf{t}, z\right\rangle_{H^{\prime}}= & \kappa(\nabla \psi, \nabla z)+\kappa \int_{\Gamma_{5}} \gamma \psi \cdot z d \Gamma \\
& +(\widetilde{\mathbf{u}} \cdot \nabla \psi, z)+(\boldsymbol{\omega} \cdot \nabla \widetilde{T}, z) \\
\mathbf{F}_{3 \mathbf{s}}(\widetilde{\mathbf{s}}) \mathbf{t}= & \boldsymbol{\omega}_{|\Gamma| \Gamma_{3}}-\mathscr{B} \boldsymbol{\varrho} \\
F_{4 \mathbf{s}}(\widetilde{\mathbf{s}}) \mathbf{t}= & \psi_{\mid \Gamma_{4}}-\tau,
\end{aligned}
$$

for all $(\mathbf{v}, z) \in \mathbb{X}$. The operator $\mathscr{B} \in \mathscr{L}\left(\widetilde{\mathbf{H}}_{00}^{1 / 2}\left(\Gamma_{2}\right), \widetilde{\mathbf{H}}_{00}^{1 / 2}\left(\Gamma \backslash \Gamma_{3}\right)\right)$ is defined by

$$
\mathscr{B} \varrho:= \begin{cases}\mathbf{0} & \text { on } \Gamma_{1}, \\ \varrho & \text { on } \Gamma_{2} .\end{cases}
$$

The following is an adaptation to the definition of regular point, given by [33, p. 50] in abstract, for optimal control problem (45).

Definition 13. Let $\widetilde{\mathbf{s}}=\left((\widetilde{\mathbf{u}}, \widetilde{T}),\left(\widetilde{\mathbf{g}}_{1}, \widetilde{g}_{2}\right)\right) \in \mathbb{H} \times \mathcal{U}_{\mathrm{ad}}$ be an admissible point for the control problem (55). We say that $\widetilde{\mathbf{s}}$ is a regular point of the operator $\mathbb{F}$, defined by (54), if

$$
\mathbb{F}_{\mathbf{s}}(\widetilde{\mathbf{s}})\left(\mathbb{W} \times \mathscr{C}\left(\widetilde{\mathbf{g}}_{1}\right) \times \mathscr{C}\left(\widetilde{g}_{2}\right)\right)=\mathbb{Y},
$$

where

$$
\begin{aligned}
& \mathscr{C}\left(\widetilde{\mathbf{g}}_{1}\right) \times \mathscr{C}\left(\widetilde{g}_{2}\right)=\left\{\left(\theta_{1}\left(\mathbf{g}_{1}-\widetilde{\mathbf{g}}_{1}\right), \theta_{2}\left(g_{2}-\widetilde{g}_{2}\right)\right): \theta_{1}\right. \\
& \left.\quad \geq 0, \quad \theta_{2} \geq 0,\left(\mathbf{g}_{1}, g_{2}\right) \in \mathscr{U}_{\mathrm{ad}}\right\} .
\end{aligned}
$$

In the following lemma, we give a condition to assure that $\widetilde{\mathbf{s}} \in \mathcal{S}_{\text {ad }}$ is a regular point for optimal control problem (45). Thereafter the existence of Lagrange multipliers is shown.
Lemma 14. Let $\widetilde{\mathbf{s}}=\left(\widetilde{\mathbf{u}}, \widetilde{T}, \widetilde{\mathbf{g}}_{1}, \widetilde{g}_{2}\right) \in \mathcal{S}_{\text {ad }}$ be a feasible solution for the optimal problem (45). If $\nu, \kappa$ are sufficiently large and $\|\gamma\|_{L^{\infty}\left(\Gamma_{5}\right)}$ is small enough, such that

$$
\begin{aligned}
& \min \left\{2 \nu-\beta C\|\mathbf{G}\|_{\infty}-C\|(\widetilde{\mathbf{u}}, \widetilde{T})\|_{\mathbb{\sharp}}, \kappa-\beta C\|\mathbf{G}\|_{\infty}\right. \\
& \left.-\kappa C\|\gamma\|_{L^{\infty}\left(\Gamma_{5}\right)}-C\|(\widetilde{\mathbf{u}}, \widetilde{T})\|_{\mathbb{\sharp}}\right\}>0,
\end{aligned}
$$

where the constant $C>0$ depends on the domain $\Omega$, then $\widetilde{\mathbf{s}}$ is a regular point.

Proof. Given $(\mathbf{a}, b, \mathbf{c}, d) \in \mathbb{Y}$, it is sufficient to show the existence of the point $\mathbf{t}=((\boldsymbol{\omega}, \psi),(\varrho, \tau)) \in \mathbb{H} \times \mathscr{U}_{\mathrm{ad}}$, such that

$$
\begin{aligned}
& 2 \nu(D(\boldsymbol{\omega}), D(\mathbf{v}))+2 \alpha \nu \int_{\Gamma_{3}} \boldsymbol{\omega} \cdot \mathbf{v} d \Gamma+(\widetilde{\mathbf{u}} \cdot \nabla \boldsymbol{\omega}, \mathbf{v}) \\
& \quad+(\boldsymbol{\omega} \cdot \nabla \widetilde{\mathbf{u}}, \mathbf{v})+\beta(\mathbf{G} \psi, \mathbf{v})=\langle\mathbf{a}, \mathbf{v}\rangle_{\widetilde{\mathbf{H}}_{\sigma}^{\prime}} \quad \forall \mathbf{v} \in \widetilde{\mathbf{H}}_{\sigma}^{1}, \\
& \kappa(\nabla \psi, \nabla z)+\kappa \int_{\Gamma_{5}} \gamma \psi \cdot z d \Gamma+(\widetilde{\mathbf{u}} \cdot \nabla \psi, z) \\
& \quad+(\boldsymbol{\omega} \cdot \nabla \widetilde{T}, z)=\langle b, z\rangle_{H^{\prime}} \quad \forall z \in H_{0}, \\
& \boldsymbol{\omega}_{|\Gamma| \Gamma_{3}}=\mathbf{c}+\mathscr{B}\left(\varrho-\widetilde{\mathbf{g}}_{1}\right), \\
& \psi_{\mid \Gamma_{4}}=d+\left(\tau-\widetilde{g}_{2}\right) .
\end{aligned}
$$

Setting $\boldsymbol{\varrho}=\widetilde{\mathbf{g}}_{1}$ and $\tau=\widetilde{\boldsymbol{g}}_{2}$, we have $\boldsymbol{\omega}_{|\Gamma| \Gamma_{3}}=\mathbf{c}$ and $\psi_{\mid \Gamma}=$ $d$; thus, from Lemma 3, there exists $\left(\boldsymbol{\omega}^{\varepsilon}, \psi^{\varepsilon}\right) \in \mathbb{H}$ such that $\boldsymbol{\omega}_{|\Gamma| \Gamma_{3}}^{\varepsilon}=\mathbf{c}, \boldsymbol{\omega}_{\mid \Gamma_{3}}^{\varepsilon}=\mathbf{0}, \psi_{\mid \Gamma_{4}}=d$, and $\psi_{\mid \Gamma_{5}}=0$. Then, rewriting the unknown $(\boldsymbol{\omega}, \psi) \in \mathbb{W}$ in the form $\boldsymbol{\omega}=\boldsymbol{\omega}^{\varepsilon}+\widehat{\boldsymbol{\omega}}$ and $\psi=\psi^{\varepsilon}+$ $\widehat{\psi}$, with $(\widehat{\boldsymbol{\omega}}, \widehat{\psi}) \in \mathbb{X}$ new unknowns functions, from system (63), we obtain the following system: Find $(\widehat{\boldsymbol{\omega}}, \widehat{\psi}) \in \mathbb{X}$ such that

$$
\begin{aligned}
& 2 \nu(D(\widehat{\boldsymbol{w}}), D(\mathbf{v}))+2 \alpha \nu \int_{\Gamma_{3}} \widehat{\boldsymbol{\omega}} \cdot \mathbf{v} d \Gamma+(\widetilde{\mathbf{u}} \cdot \nabla \widehat{\boldsymbol{w}}, \mathbf{v}) \\
& \quad+(\widehat{\boldsymbol{\omega}} \cdot \nabla \widetilde{\mathbf{u}}, \mathbf{v})+\beta(\mathbf{G} \widehat{\psi}, \mathbf{v})=\langle\widehat{\mathbf{a}}, \mathbf{v}\rangle_{\widetilde{\mathbf{H}}_{\sigma}^{\prime}} \quad \forall \mathbf{v} \in \widetilde{\mathbf{H}}_{\sigma}^{1}, \\
& \kappa(\nabla \widehat{\psi}, \nabla z)+\kappa \int_{\Gamma_{5}} \gamma \widehat{\psi} \cdot z d \Gamma+(\widetilde{\mathbf{u}} \cdot \nabla \widehat{\psi}, z) \\
& \quad+(\widehat{\boldsymbol{\omega}} \cdot \nabla \widetilde{T}, z)=\langle\widehat{b}, z\rangle_{H^{\prime}} \quad \forall z \in H_{0},
\end{aligned}
$$

where

$$
\begin{aligned}
\langle\widehat{\mathbf{a}}, \mathbf{v}\rangle_{\widetilde{\mathbf{H}}_{\sigma}^{\prime}}:= & -2 \nu\left(D\left(\boldsymbol{\omega}^{\varepsilon}\right), D(\mathbf{v})\right)-\left(\widetilde{\mathbf{u}} \cdot \nabla \boldsymbol{\omega}^{\varepsilon}, \mathbf{v}\right) \\
& -\left(\boldsymbol{\omega}^{\varepsilon} \cdot \nabla \widetilde{\mathbf{u}}, \mathbf{v}\right)-\beta\left(\mathbf{G} \psi^{\varepsilon}, \mathbf{v}\right) \\
& +\langle\mathbf{a}, \mathbf{v}\rangle_{\widetilde{\mathbf{H}}_{\sigma}^{\prime}} \\
\langle\widehat{b}, z\rangle_{H^{\prime}}:= & -\kappa\left(\nabla \psi^{\varepsilon}, \nabla z\right)-\left(\widetilde{\mathbf{u}} \cdot \nabla \psi^{\varepsilon}, z\right) \\
& -\left(\boldsymbol{\omega}^{\varepsilon} \cdot \nabla \widetilde{T}, z\right)+\langle b, z\rangle_{H^{\prime}} .
\end{aligned}
$$


In order to prove the existence of a solution for system (64) we define the bilinear form $a: \mathbb{X} \times \mathbb{X} \rightarrow \mathbb{R}$ by

$$
\begin{aligned}
a((\widehat{\boldsymbol{\omega}}, \widehat{\psi}),(\mathbf{v}, z)):= & 2 \nu(D(\widehat{\boldsymbol{\omega}}), D(\mathbf{v})) \\
& +2 \alpha \nu \int_{\Gamma_{3}} \widehat{\boldsymbol{\omega}} \cdot \mathbf{v} d \Gamma+(\widetilde{\mathbf{u}} \cdot \nabla \widehat{\boldsymbol{\omega}}, \mathbf{v}) \\
& +(\widehat{\boldsymbol{\omega}} \cdot \nabla \widetilde{\mathbf{u}}, \mathbf{v})+\beta(\mathbf{G} \widehat{\psi}, \mathbf{v}) \\
& +\kappa(\nabla \widehat{\psi}, \nabla z)+(\widetilde{\mathbf{u}} \cdot \nabla \widehat{\psi}, z) \\
& +(\widehat{\boldsymbol{\omega}} \cdot \nabla \widetilde{T}, z)+\kappa \int_{\Gamma_{5}} \gamma \widehat{\psi} \cdot z d \Gamma,
\end{aligned}
$$

and the linear and continuous functional $\mathscr{F}: \mathbb{X} \rightarrow \mathbb{R}$ by

$$
\mathscr{F}(\mathbf{v}, z):=\langle\widehat{\mathbf{a}}, \mathbf{v}\rangle_{\widetilde{\mathbf{H}}_{\sigma}^{\prime}}+\langle\widehat{b}, z\rangle_{H^{\prime}} .
$$

Thus, from (66)-(67), system (64) is equivalent to the following. Find $(\widehat{\boldsymbol{\omega}}, \widehat{\psi}) \in \mathbb{X}$ such that

$$
a((\widehat{\boldsymbol{\omega}}, \widehat{\psi}),(\mathbf{v}, z))=\mathscr{F}(\mathbf{v}, z) \quad \forall(\mathbf{v}, z) \in \mathbb{X} .
$$

We notice that the form $a(\cdot, \cdot)$ is continuous. Now, by taking $(\mathbf{v}, z)=(\widehat{\boldsymbol{w}}, \widehat{\psi})$ in $(66)$, we have

$$
\begin{aligned}
a((\widehat{\boldsymbol{\omega}}, \widehat{\psi}),(\widehat{\boldsymbol{\omega}}, \widehat{\psi})) \geq & 2 v\|\widehat{\boldsymbol{\omega}}\|_{\widetilde{\mathbf{H}}_{\sigma}^{1}}^{2}+(\widehat{\boldsymbol{\omega}} \cdot \nabla \widetilde{\mathbf{u}}, \widehat{\boldsymbol{\omega}}) \\
& +\beta(\mathbf{G} \widehat{\psi}, \widehat{\boldsymbol{\omega}})+\kappa\|\psi\|_{H_{0}}^{2} \\
& +(\widehat{\boldsymbol{\omega}} \cdot \nabla \widetilde{T}, \widehat{\psi})+\kappa \int_{\Gamma_{5}} \gamma \widehat{\psi} \cdot \widehat{\psi} d \Gamma .
\end{aligned}
$$

By applying the Hölder and Young inequalities to the terms in the right-hand side of (69), we have

$$
\begin{aligned}
& |(\widehat{\boldsymbol{\omega}} \cdot \nabla \widetilde{\mathbf{u}}, \widehat{\boldsymbol{\omega}})+(\widehat{\boldsymbol{\omega}} \cdot \nabla \widetilde{T}, \widehat{\psi})| \\
& \quad \leq C\|\widetilde{\mathbf{u}}\|_{\mathbf{H}_{\sigma}^{1}}\|\widehat{\boldsymbol{\omega}}\|_{\widetilde{\mathbf{H}}_{\sigma}^{1}}^{2}+C\|\widetilde{T}\|_{H^{1}}\left(\|\widehat{\boldsymbol{\omega}}\|_{\widetilde{\mathbf{H}}_{\sigma}^{1}}^{2}+\|\widehat{\psi}\|_{H_{0}}^{2}\right), \\
& \quad|\beta(\mathbf{G} \widehat{\psi}, \widehat{\boldsymbol{\omega}})| \leq \beta\|\mathbf{G}\|_{\infty}\|\widehat{\psi}\|\|\widehat{\boldsymbol{\omega}}\| \\
& \quad \leq \beta C\|\mathbf{G}\|_{\infty}\left(\|\widehat{\boldsymbol{\omega}}\|_{\widetilde{\mathbf{H}}_{\sigma}^{1}}^{2}+\|\widehat{\psi}\|_{H_{0}}^{2}\right) \\
& \left|\mathcal{\kappa} \int_{\Gamma_{5}} \gamma \widehat{\psi} \cdot \widehat{\psi} d \Gamma\right| \leq \kappa\|\gamma\|_{L^{\infty}\left(\Gamma_{5}\right)}\|\widehat{\psi}\|_{L^{2}\left(\Gamma_{5}\right)}^{2} \\
& \quad \leq \kappa C\|\gamma\|_{L^{\infty}\left(\Gamma_{5}\right)}\|\widehat{\psi}\|_{H_{0}}^{2} .
\end{aligned}
$$

Then, by using the last inequalities in (69), we have

$$
\begin{aligned}
& a((\widehat{\boldsymbol{\omega}}, \widehat{\psi}),(\widehat{\boldsymbol{\omega}}, \widehat{\psi})) \\
& \geq\left(2 v-C\|\widetilde{\mathbf{u}}\|_{\mathbf{H}_{\sigma}^{1}}-C\|\widetilde{T}\|_{H^{1}}-\beta C\|\mathbf{G}\|_{\infty}\right)\|\widehat{\boldsymbol{\omega}}\|_{\widetilde{\mathbf{H}}_{\sigma}^{1}}^{2} \\
& \quad+\left(\kappa-C\|\widetilde{T}\|_{H^{1}}-\beta C\|\mathbf{G}\|_{\infty}-\kappa C\|\gamma\|_{L^{\infty}\left(\Gamma_{5}\right)}\right)\|\widehat{\psi}\|_{H_{0}}^{2} \\
& \geq \bar{\delta}\|(\widehat{\boldsymbol{\omega}}, \widehat{\psi})\|_{\mathbb{X}}^{2},
\end{aligned}
$$

where, by hypotheses, $\bar{\delta}:=\min \left\{2 \nu-\beta C\|\mathbf{G}\|_{\infty}-C\|(\widetilde{\mathbf{u}}, \widetilde{T})\|_{\mathbb{\sharp}}, \kappa-\right.$ $\left.\beta C\|\mathbf{G}\|_{\infty}-\kappa C\|\gamma\|_{L^{\infty}\left(\Gamma_{5}\right)}-\|C(\widetilde{\mathbf{u}}, \widetilde{T})\|_{\mathbb{\sharp}}\right\}>0$. Thus, we obtain that the form $a(\cdot, \cdot)$ is $\mathbb{X}$-coercive; therefore, from (68), (71), and the Lax-Milgram theorem we conclude the existence of a unique $(\widehat{\boldsymbol{\omega}}, \widehat{\psi}) \in \mathbb{X}$ solution of system (64), and, consequently, we obtain that $(\boldsymbol{\omega}, \psi) \in \mathbb{W}$ is solution of (63).

In the following result we prove the existence of Lagrange multipliers provided that a local optimal solution $\widetilde{\mathbf{s}}=$ $\left(\widetilde{\mathbf{u}}, \widetilde{T}, \widetilde{\mathbf{g}}_{1}, \widetilde{g}_{2}\right) \in \mathcal{S}_{\mathrm{ad}}$ verifies the regular point condition provided by Lemma 14 .

Theorem 15. Let $\widetilde{\mathbf{s}}=\left(\widetilde{\mathbf{u}}, \widetilde{T}, \widetilde{\mathbf{g}}_{1}, \widetilde{g}_{2}\right) \in \mathcal{S}_{\text {ad }}$ be a local optimal solution for the optimal control problem (45) satisfying the regular point condition given in (62). Then, there exist Lagrange multipliers $((\boldsymbol{\lambda}, \eta), \boldsymbol{\chi}, \vartheta) \in \mathbb{} \times \times \widetilde{\mathbf{H}}_{00}^{-1 / 2}\left(\Gamma \backslash \Gamma_{3}\right) \times H_{00}^{-1 / 2}\left(\Gamma_{4}\right)$ such that for all $((\boldsymbol{\omega}, \psi), \varrho, \tau) \in \mathbb{U} \times \mathscr{C}\left(\widetilde{\mathbf{g}}_{1}\right) \times \mathscr{C}\left(\widetilde{g}_{2}\right)$ it holds that

$$
\begin{aligned}
(\widetilde{\mathbf{u}}- & \left.\mathbf{u}_{d}, \boldsymbol{\omega}\right)+\left(\widetilde{T}-T_{d}, \psi\right)+b_{1} \int_{\Gamma_{2}} \widetilde{\mathbf{g}}_{1} \cdot \varrho d \Gamma \\
& +b_{2} \int_{\Gamma_{4}} \tilde{g}_{2} \cdot \tau d \Gamma-2 \nu(D(\boldsymbol{\omega}), D(\boldsymbol{\lambda})) \\
& -2 \alpha \nu \int_{\Gamma_{3}} \boldsymbol{\omega} \cdot \boldsymbol{\lambda} d \Gamma-(\widetilde{\mathbf{u}} \cdot \nabla \boldsymbol{\omega}, \boldsymbol{\lambda})-(\boldsymbol{\omega} \cdot \nabla \widetilde{\mathbf{u}}, \boldsymbol{\lambda}) \\
& -\beta(\mathbf{G} \psi, \boldsymbol{\lambda})-\kappa(\nabla \psi, \nabla \eta)-(\widetilde{\mathbf{u}} \cdot \nabla \psi, \eta) \\
& -(\boldsymbol{\omega} \cdot \nabla \widetilde{T}, \eta)-\kappa \int_{\Gamma_{5}} \gamma \psi \cdot \eta d \Gamma \\
& -\langle\boldsymbol{\chi}, \boldsymbol{\omega}-\mathscr{B} \boldsymbol{\varrho}\rangle_{\Gamma \backslash \Gamma_{3}}-\langle\vartheta, \psi-\tau\rangle_{\Gamma_{4}} \geq 0,
\end{aligned}
$$

where $\mathscr{C}\left(\widetilde{\mathbf{g}}_{1}\right) \times \mathscr{C}\left(\widetilde{g}_{2}\right)$ is defined in (61).

Proof. Since $\widetilde{\mathbf{s}}=\left(\widetilde{\mathbf{u}}, \widetilde{T}, \widetilde{\mathbf{g}}_{1}, \widetilde{g}_{2}\right) \in \mathcal{S}_{\text {ad }}$ satisfies the regular point condition (62), then there exist Lagrange multipliers $((\lambda, \eta), \boldsymbol{\chi}, \vartheta) \in \mathbb{X} \times \widetilde{\mathbf{H}}_{00}^{-1 / 2}\left(\Gamma \backslash \Gamma_{3}\right) \times H_{00}^{-1 / 2}\left(\Gamma_{4}\right)$ such that

$$
\begin{aligned}
J_{\mathbf{s}}(\widetilde{\mathbf{s}}) \mathbf{t} & -\left\langle\mathbf{F}_{1 \mathbf{s}}(\widetilde{\mathbf{s}}) \mathbf{t}, \lambda\right\rangle_{\widetilde{\mathbf{H}}_{\sigma}^{\prime}}-\left\langle F_{2 \mathbf{s}}(\widetilde{\mathbf{s}}) \mathbf{t}, \eta\right\rangle_{H^{\prime}} \\
& -\left\langle\boldsymbol{\chi}, \mathbf{F}_{3 \mathbf{s}}(\widetilde{\mathbf{s}}) \mathbf{t}\right\rangle_{\Gamma \backslash \Gamma_{3}}-\left\langle\vartheta, F_{4 \mathbf{s}}(\widetilde{\mathbf{s}}) \mathbf{t}\right\rangle_{\Gamma_{4}} \geq 0,
\end{aligned}
$$

for all $\mathbf{t}=((\boldsymbol{\omega}, \psi), \varrho, \tau) \in \mathbb{U} \times \mathscr{C}\left(\widetilde{\mathbf{g}}_{1}\right) \times \mathscr{C}\left(\widetilde{g}_{2}\right)$. Thus, the proof follows from (57), (58), and (59).

4.3. Optimality System. In this subsection we derive the equations that are satisfied by the Lagrange multipliers $((\lambda, \eta), \boldsymbol{\chi}, \vartheta) \in \mathbb{X} \times \widetilde{\mathbf{H}}_{00}^{-1 / 2}\left(\Gamma \backslash \Gamma_{3}\right) \times H_{00}^{-1 / 2}\left(\Gamma_{4}\right)$ provided by Theorem 15 .

Theorem 16. Under the conditions of Theorem 15, the Lagrange multipliers $(\lambda, \eta, \chi, \vartheta)$ satisfy the following adjoint equations

$$
\begin{aligned}
& -v \Delta \boldsymbol{\lambda}-\widetilde{\mathbf{u}} \cdot \nabla \boldsymbol{\lambda}+\nabla^{T} \boldsymbol{\lambda} \cdot \widetilde{\mathbf{u}}+\nabla^{T} \eta \cdot \widetilde{T}=\widetilde{\mathbf{u}}-\mathbf{u}_{d} \quad \text { in } \widetilde{\mathbf{H}}_{\sigma}^{\prime}, \\
& -\kappa \Delta \eta-\widetilde{\mathbf{u}} \cdot \nabla \eta+\beta \mathbf{G} \cdot \boldsymbol{\lambda}=\widetilde{T}-T_{d} \text { in } H^{\prime},
\end{aligned}
$$




$$
\begin{array}{rlrl}
\operatorname{div} \boldsymbol{\lambda} & =0 & & \text { in } \Omega, \\
\nu(\nabla \boldsymbol{\lambda}) \mathbf{n}=\mathbf{0} & & \text { on } \Gamma \backslash \Gamma_{3}, \\
\nu(\nabla \boldsymbol{\lambda}) \mathbf{n}+\alpha \boldsymbol{\lambda}=\mathbf{0} & & \text { on } \Gamma_{3}, \\
(\nabla \eta) \mathbf{n} & =0 & & \text { on } \Gamma_{4}, \\
(\nabla \eta) \mathbf{n}+\kappa(\gamma \eta) & =0 & & \text { on } \Gamma_{5}, \\
\chi & =\mathbf{0} & & \text { on } \Gamma \backslash \Gamma_{3}, \\
\vartheta & =0 & & \text { on } \Gamma_{4},
\end{array}
$$

and the optimality conditions

$$
\begin{aligned}
& \left\langle b_{1} \widetilde{\mathbf{g}}_{1}+\chi, \mathbf{g}_{1}-\widetilde{\mathbf{g}}_{1}\right\rangle_{\Gamma_{2}} \geq 0, \\
& \left\langle b_{2} \widetilde{g}_{2}+\vartheta, g_{2}-\widetilde{g}_{2}\right\rangle_{\Gamma_{4}} \geq 0 .
\end{aligned}
$$

Proof. In fact, from (72), taking $(\psi, \varrho, \tau)=(0, \mathbf{0}, 0)$, we have

$$
\begin{aligned}
(\widetilde{\mathbf{u}}- & \left.\mathbf{u}_{d}, \boldsymbol{\omega}\right)-2 \nu(D(\boldsymbol{\omega}), D(\boldsymbol{\lambda}))-2 \alpha \nu \int_{\Gamma_{3}} \boldsymbol{\omega} \cdot \lambda d \Gamma \\
& -(\widetilde{\mathbf{u}} \cdot \nabla \boldsymbol{\omega}, \boldsymbol{\lambda})-(\boldsymbol{\omega} \cdot \nabla \widetilde{\mathbf{u}}, \boldsymbol{\lambda})-(\boldsymbol{\omega} \cdot \nabla \widetilde{T}, \eta) \\
& -\langle\boldsymbol{\chi}, \boldsymbol{\omega}\rangle_{\Gamma \backslash \Gamma_{3}}=0 \quad \forall \boldsymbol{\omega} \in \mathbf{H}_{\sigma}^{1},
\end{aligned}
$$

and integrating by parts, for all $\boldsymbol{\omega} \in \mathbf{H}_{\sigma}^{1}$, we obtain

$$
\begin{aligned}
(\widetilde{\mathbf{u}}- & \left.\mathbf{u}_{d}, \boldsymbol{\omega}\right)+\nu(\Delta \boldsymbol{\lambda}, \boldsymbol{\omega})-2 \nu \int_{\Gamma}[(\nabla \boldsymbol{\lambda}) \mathbf{n}] \cdot \boldsymbol{\omega} d \Gamma \\
& -2 \alpha \nu \int_{\Gamma_{3}} \boldsymbol{\lambda} \cdot \boldsymbol{\omega} d \Gamma+(\widetilde{\mathbf{u}} \cdot \nabla \boldsymbol{\lambda}, \boldsymbol{\omega})-\left(\nabla^{T} \boldsymbol{\lambda} \cdot \mathbf{u}, \boldsymbol{\omega}\right) \\
& -\left(\nabla^{T} \eta \cdot \widetilde{T}, \boldsymbol{\omega}\right)-\langle\boldsymbol{\chi}, \boldsymbol{\omega}\rangle_{\Gamma \backslash \Gamma_{3}}=0 .
\end{aligned}
$$

Now, taking $(\boldsymbol{\omega}, \varrho, \tau)=(\mathbf{0}, \mathbf{0}, 0)$ in $(72)$, we have

$$
\begin{gathered}
\left(\widetilde{T}-T_{d}, \psi\right)-\beta(\mathbf{G} \psi, \lambda)-\kappa(\nabla \psi, \nabla \eta)-(\widetilde{\mathbf{u}} \cdot \nabla \psi, \eta) \\
-\kappa \int_{\Gamma_{5}} \gamma \psi \cdot \eta d \Gamma-\langle\vartheta, \psi\rangle_{\Gamma_{4}}=0,
\end{gathered}
$$

for all $\psi \in H^{1}(\Omega)$, and integrating by parts, for all $\psi \in H^{1}(\Omega)$, we obtain

$$
\begin{aligned}
(\widetilde{T}- & \left.T_{d}, \psi\right)+\kappa(\Delta \eta, \psi)-\kappa \int_{\Gamma}[(\nabla \eta) \mathbf{n}] \cdot \psi d \Gamma \\
& +(\widetilde{\mathbf{u}} \cdot \nabla \eta, \psi)-\beta(\mathbf{G} \cdot \lambda, \psi)-\kappa \int_{\Gamma_{5}} \gamma \eta \cdot \psi d \Gamma \\
& -\langle\vartheta, \psi\rangle_{\Gamma_{4}}=0 .
\end{aligned}
$$

Since $(\boldsymbol{\omega}, \psi) \in \mathbb{U}$ is arbitrary, then from (82) and (84), we obtain system (74)-(79).

Now, from $(72)$, when $(\boldsymbol{\omega}, \psi)=(\mathbf{0}, 0)$, we obtain

$$
\begin{aligned}
& b_{1} \int_{\Gamma_{2}} \widetilde{\mathbf{g}}_{1} \cdot \varrho d \Gamma+b_{2} \int_{\Gamma_{4}} \widetilde{g}_{2} \cdot \tau d \Gamma+\langle\boldsymbol{\chi}, \mathscr{B} \boldsymbol{\varrho}\rangle_{\Gamma \backslash \Gamma_{3}} \\
& \quad+\langle\vartheta, \tau\rangle_{\Gamma_{4}} \geq 0 .
\end{aligned}
$$

Taking $\boldsymbol{\varrho}=\mathbf{g}_{1}-\widetilde{\mathbf{g}}_{1}, \tau=g_{2}-\widetilde{g}_{2}$, and taking into account the definition of operator $\mathscr{B}$ given in (59), the last inequality implies

$$
\left\langle b_{1} \widetilde{\mathbf{g}}_{1}+\chi, \mathbf{g}_{1}-\widetilde{\mathbf{g}}_{1}\right\rangle_{\Gamma_{2}}+\left\langle b_{2} \widetilde{g}_{2}+\vartheta, g_{2}-\widetilde{g}_{2}\right\rangle_{\Gamma_{4}} \geq 0
$$

thus the optimality conditions (80) follow.

Summarizing, the state equations described in (1)-(4), the adjoint equations given in (75)-(79), and the optimality conditions obtained in (80) form the optimality system of the optimal control problem (45).

Remark 17. Since the set of controls $\mathcal{U}_{a d}$ is convex, then from (80) we deduce

$$
\begin{aligned}
& \widetilde{\mathbf{g}}_{1}=\underset{\mathcal{U}_{1}}{\operatorname{Proj}}\left(-\frac{\chi}{b_{1}}\right) \text { on } \Gamma_{2}, \\
& \widetilde{g}_{2}=\underset{\mathcal{u}_{2}}{\operatorname{Proj}}\left(-\frac{\vartheta}{b_{2}}\right) \text { on } \Gamma_{4} .
\end{aligned}
$$

\section{Second-Order Sufficient Condition}

In this section, we will discuss sufficient conditions for $\widetilde{\mathbf{s}}=$ $\left(\widetilde{\mathbf{u}}, \widetilde{T}, \widetilde{\mathbf{g}}_{1}, \widetilde{g}_{2}\right) \in \mathcal{S}_{\text {ad }}$ being a local optimal solution for control problem (45). We will establish a $\mathbb{U} \times \mathcal{U}_{\text {ad }}$-coercivity condition on the second derivative of the Lagrange functional $\mathscr{L}$ given in (56) associated with problem (45) in order to assure that an admissible point $\widetilde{\mathbf{s}}$ is a local optimal solution. Due to Lemmas 11 and 12, the functional $\mathscr{L}$ is Fréchet differentiable with respect to the point $\mathbf{s}=\left((\mathbf{u}, T),\left(\mathbf{g}_{1}, g_{2}\right)\right) \in \mathbb{M} \times \mathscr{U}_{\mathrm{ad}}$. Then, we can obtain the fact that that the Lagrange multiplier $\widetilde{\phi}=((\lambda, \eta), \chi, \vartheta) \in \mathbb{X} \times \widetilde{\mathbf{H}}_{00}^{-1 / 2}\left(\Gamma \backslash \Gamma_{3}\right) \times H_{00}^{-1 / 2}\left(\Gamma_{4}\right)$, given by Theorem 15 , for $\mathbf{x}=(\mathbf{u}, T) \in \mathbb{M}$, satisfies $\mathscr{L}_{\mathbf{x}}(\widetilde{\mathbf{s}}, \widetilde{\phi})(\boldsymbol{\omega}, \psi)=0$; that is,

$$
\begin{aligned}
(\widetilde{\mathbf{u}}- & \left.\mathbf{u}_{d}, \boldsymbol{\omega}\right)+\left(\widetilde{T}-T_{d}, \psi\right)-2 \nu(D(\boldsymbol{\omega}), D(\boldsymbol{\lambda})) \\
& -2 \alpha \nu \int_{\Gamma_{3}} \boldsymbol{\omega} \cdot \lambda d \Gamma-(\widetilde{\mathbf{u}} \cdot \nabla \boldsymbol{\omega}, \boldsymbol{\lambda})-(\boldsymbol{\omega} \cdot \nabla \widetilde{\mathbf{u}}, \boldsymbol{\lambda}) \\
& -\beta(\mathbf{G} \psi, \boldsymbol{\lambda})-\kappa(\nabla \psi, \nabla \eta)-(\widetilde{\mathbf{u}} \cdot \nabla \psi, \eta) \\
& -(\boldsymbol{\omega} \cdot \nabla \widetilde{T}, \eta)-\kappa \int_{\Gamma_{5}} \gamma \psi \cdot \eta d \Gamma-\langle\boldsymbol{\chi}, \boldsymbol{\omega}\rangle_{\Gamma \backslash \Gamma_{3}} \\
& -\langle\vartheta, \psi\rangle_{\Gamma_{4}}=0,
\end{aligned}
$$

for all $(\boldsymbol{\omega}, \psi) \in \mathbb{H}$; this equation is called the Lagrange-Euler equation. In this section we will follow the ideas of $[20,30]$ (see also [28]).

Lemma 18. Let $\widetilde{\mathbf{s}}=\left(\widetilde{\mathbf{u}}, \widetilde{T}, \widetilde{\mathbf{g}}_{1}, \widetilde{g}_{2}\right) \in \mathcal{S}_{a d}$ be an admissible point for the optimal control problem (45). If $\nu, \kappa$ are sufficiently large and $\|\gamma\|_{L^{\infty}\left(\Gamma_{5}\right)}$ is small enough, such that

$$
\begin{aligned}
\widehat{\delta}: & =\min \left\{v-\beta C\|\mathbf{G}\|_{\infty}-C\|(\widetilde{\mathbf{u}}, \widetilde{T})\|_{\mathbb{\square}}, \frac{\kappa}{2}\right. \\
& \left.-\beta C\|\mathbf{G}\|_{\infty}-\kappa C\|\gamma\|_{L^{\infty}\left(\Gamma_{5}\right)}-C\|(\widetilde{\mathbf{u}}, \widetilde{T})\|_{\mathbb{⿴}}\right\}>0,
\end{aligned}
$$


then the Lagrange multiplier $(\lambda, \eta) \in \mathbb{X}$ satisfies

$$
\|(\lambda, \eta)\|_{\mathbb{X}}^{2} \leq \frac{1}{\widehat{\delta}} \widetilde{C} \mathcal{N}(\widetilde{\mathbf{u}}, \widetilde{T}),
$$

where $\mathcal{N}:=\left\|\widetilde{\mathbf{u}}-\mathbf{u}_{2}\right\|^{2}+\left\|\widetilde{T}-T_{d}\right\|^{2}$ and $\widetilde{C}:=C \max \{1 / 4 \nu, 1 / 2 \kappa\}$, with $C$ being a positive constant depending only on $\Omega$.

Proof. Setting $(\boldsymbol{\omega}, \psi)=(\boldsymbol{\lambda}, \tau) \in \mathbb{X}$ in (88), we have

$$
\begin{aligned}
2 \nu\|\lambda\|_{\widetilde{\mathbf{H}}_{\sigma}^{1}}^{2}+\kappa\|\eta\|_{H_{0}}^{2} \leq & \left(\widetilde{\mathbf{u}}-\mathbf{u}_{d}, \lambda\right)+\left(\widetilde{T}-T_{d}, \eta\right) \\
& -(\lambda \cdot \nabla \widetilde{\mathbf{u}}, \lambda)-\beta(\mathbf{G} \eta, \lambda) \\
& -(\lambda \cdot \nabla \widetilde{T}, \eta)-\kappa \int_{\Gamma_{5}} \gamma \eta \cdot \eta d \Gamma .
\end{aligned}
$$

Applying the Hölder and Young inequalities in terms of the right side of (91), we obtain

$$
\begin{aligned}
& \left|\left(\widetilde{\mathbf{u}}-\mathbf{u}_{d}, \lambda\right)\right| \leq\left\|\widetilde{\mathbf{u}}-\mathbf{u}_{d}\right\|\|\lambda\| \\
& \quad \leq \frac{C}{4 \nu}\left\|\widetilde{\mathbf{u}}-\mathbf{u}_{d}\right\|^{2}+\nu\|\lambda\|_{\widetilde{\mathbf{H}}_{\sigma}^{1}}^{2}, \\
& \left|\left(\widetilde{T}-T_{d}, \eta\right)\right| \leq\left\|\widetilde{T}-T_{d}\right\|\|\eta\| \\
& \quad \leq \frac{C}{2 \kappa}\left\|\widetilde{T}-T_{d}\right\|^{2}+\frac{\kappa}{2}\|\eta\|_{H_{0}}^{2}, \\
& |\beta(\mathbf{G} \eta, \lambda)| \leq \beta\|\mathbf{G}\|_{\infty}\|\eta\|\|\lambda\| \\
& \quad \leq \beta C\|\mathbf{G}\|_{\infty}\left(\|\lambda\|_{\widetilde{\mathbf{H}}_{\sigma}^{1}}^{2}+\|\eta\|_{H_{0}}^{2}\right), \\
& \quad|(\lambda \cdot \nabla \widetilde{\mathbf{u}}, \lambda)+(\lambda \cdot \nabla \widetilde{T}, \eta)| \\
& \quad \leq C\|\widetilde{\mathbf{u}}\|_{\mathbf{H}_{\sigma}^{1}}\|\lambda\|_{\widetilde{\mathbf{H}}_{\sigma}^{1}}^{2}+C\|\widetilde{T}\|_{H^{1}}\left(\|\lambda\|_{\widetilde{\mathbf{H}}_{\sigma}^{1}}^{2}+\|\eta\|_{H_{0}}^{2}\right) \\
& \quad \leq C\|(\widetilde{\mathbf{u}}, \widetilde{T})\|_{\mathbb{H}^{-}}\|\lambda\|_{\widetilde{\mathbf{H}}_{\sigma}^{1}}^{2}+C\|(\widetilde{\mathbf{u}}, \widetilde{T})\|_{\mathbb{H}}\|\eta\|_{H_{0}}^{2}, \\
& \left|\kappa \int_{\Gamma_{5}} \gamma \eta \cdot \eta d \Gamma\right| \leq \kappa\|\|_{L^{\infty}\left(\Gamma_{5}\right)}\|\eta\|_{L^{2}\left(\Gamma_{5}\right)}^{2} \\
& \quad \leq \kappa C\|\gamma\|_{L^{\infty}\left(\Gamma_{5}\right)}\|\eta\|_{H_{0}}^{2} \cdot
\end{aligned}
$$

Then, by replacing the last inequalities in (91), we have

$$
\begin{aligned}
& \left(\nu-\beta C\|\mathbf{G}\|_{\infty}-C\|(\widetilde{\mathbf{u}}, \widetilde{T})\|_{\mathbb{H}}\right)\|\lambda\|_{\widetilde{\mathbf{H}}_{\sigma}^{1}}^{2} \\
& +\left(\frac{\kappa}{2}-\beta C\|\mathbf{G}\|_{\infty}-\kappa C\|\gamma\|_{L^{\infty}\left(\Gamma_{5}\right)}-C\|(\widetilde{\mathbf{u}}, \widetilde{T})\|_{\mathbb{H}}\right) \\
& \cdot\|\eta\|_{H_{0}}^{2} \leq \frac{C}{4 \nu}\left\|\widetilde{\mathbf{u}}-\mathbf{u}_{d}\right\|^{2}+\frac{C}{2 \kappa}\left\|\widetilde{T}-T_{d}\right\|^{2} ;
\end{aligned}
$$

since, by hypothesis,

$$
\begin{aligned}
& \widehat{\delta}:=\min \left\{v-\beta C\|\mathbf{G}\|_{\infty}-C\|(\widetilde{\mathbf{u}}, \widetilde{T})\|_{\mathbb{H}}, \frac{\kappa}{2}\right. \\
& \left.-\beta C\|\mathbf{G}\|_{\infty}-\kappa C\|\gamma\|_{L^{\infty}\left(\Gamma_{5}\right)}-C\|(\widetilde{\mathbf{u}}, \widetilde{T})\|_{\mathbb{H}}\right\}>0,
\end{aligned}
$$

then, from the last inequality, we conclude the proof.
Theorem 19. Let $\widetilde{\mathbf{s}}=\left(\widetilde{\mathbf{u}}, \widetilde{T}, \widetilde{\mathbf{g}}_{1}, \widetilde{g}_{2}\right) \in \mathcal{S}_{a d}$ be an admissible point for the optimal control problem (45), $v, \kappa$ be sufficiently large, and $\|\gamma\|_{L^{\infty}\left(\Gamma_{5}\right)}$ be small enough, such that

$$
\begin{aligned}
\widehat{\delta} & =\min \left\{\nu-\beta C\|\mathbf{G}\|_{\infty}-C\|(\widetilde{\mathbf{u}}, \widetilde{T})\|_{\mathbb{H}}, \frac{\kappa}{2}-\beta C\|\mathbf{G}\|_{\infty}\right. \\
& \left.-\kappa C\|\gamma\|_{L^{\infty}\left(\Gamma_{5}\right)}-C\|(\widetilde{\mathbf{u}}, \widetilde{T})\|_{\mathbb{H}}\right\}>0 .
\end{aligned}
$$

If $\left(\widetilde{C}^{2} C^{2} / \widehat{\delta} \Pi^{2}\right) \mathcal{N}(\widetilde{\mathbf{u}}, \widetilde{T})<1$, where $\Pi:=\min \left\{C b_{1} \widehat{\delta}^{2} /(1+\right.$ $\left.\widehat{\delta})^{2}, C b_{2} \widehat{\delta}^{2} /(1+\widehat{\delta})^{2}\right\}$ and $\mathcal{N}(\widetilde{\mathbf{u}}, \widetilde{T}), \widetilde{C}$ are given in Lemma 18 , then there exists a constant $\mathscr{K}>0$ such that

$$
\mathscr{L}_{\mathbf{s s}}(\widetilde{\mathbf{s}}, \widetilde{\phi})(\mathbf{t}, \mathbf{t}) \geq \mathscr{K}\|\mathbf{t}\|_{\mathbb{W} \times \mathscr{U}_{a d}}^{2},
$$

for all $\mathbf{t} \in \operatorname{ker}\left(\mathbb{F}_{\mathbf{s}}(\mathbf{s})\right)$, with $\widetilde{\boldsymbol{\phi}}=((\boldsymbol{\lambda}, \eta), \boldsymbol{\chi}, \vartheta) \in \mathbb{X} \times \widetilde{\mathbf{H}}_{00}^{-1 / 2}(\Gamma \backslash$ $\left.\Gamma_{3}\right) \times H_{00}^{-1 / 2}\left(\Gamma_{4}\right)$ given by Theorem 15 . Consequently, the point $\widetilde{\mathbf{s}}$ is a local optimal solution for problem (45).

Proof. Let $\mathbf{t}=((\boldsymbol{\omega}, \psi),(\boldsymbol{\varrho}, \tau)) \in \mathbb{U} \times \mathcal{U}_{\mathrm{ad}}$. Then, the second derivative of Lagrangian given in (56), with respect to $s$ at the point $\widetilde{\mathbf{s}}$ in $(\mathbf{t}, \mathbf{t})$, is

$$
\begin{aligned}
\mathscr{L}_{\mathbf{s s}}(\widetilde{\mathbf{s}}, \widetilde{\boldsymbol{\phi}})(\mathbf{t}, \mathbf{t})= & \|\boldsymbol{\omega}\|^{2}+\|\psi\|^{2}+b_{1}\|\boldsymbol{\rho}\|_{\mathbf{H}^{1 / 2}\left(\Gamma_{2}\right)}^{2} \\
& +b_{2}\|\tau\|_{H^{1 / 2}\left(\Gamma_{4}\right)}^{2}-2(\boldsymbol{\omega} \cdot \nabla \boldsymbol{\omega}, \lambda) \\
& -2(\boldsymbol{\omega} \cdot \nabla \psi, \eta) .
\end{aligned}
$$

By applying the Hölder and Young inequalities, we can obtain

$$
\begin{aligned}
\mathscr{L}_{\mathbf{s s}}(\widetilde{\mathbf{s}}, \widetilde{\boldsymbol{\phi}})(\mathbf{t}, \mathbf{t}) \geq & \|\boldsymbol{\omega}\|^{2}+\|\psi\|^{2}+b_{1}\|\boldsymbol{\varrho}\|_{\mathbf{H}^{1 / 2}\left(\Gamma_{2}\right)}^{2} \\
& +b_{2}\|\tau\|_{H^{1 / 2}\left(\Gamma_{4}\right)}^{2} \\
& -C\left(\|\lambda\|_{\widetilde{H}_{\sigma}^{1}}+\|\eta\|_{H_{0}}\right)\|(\boldsymbol{\omega}, \psi)\|_{\mathbb{\sharp}}^{2} .
\end{aligned}
$$

On the other hand, if $\mathbf{t} \in \operatorname{ker}\left(\mathbb{F}_{\mathbf{s}}(\widetilde{\mathbf{s}})\right)$, then, from (58)-(59), we obtain

$$
\begin{aligned}
& 2 \nu(D(\boldsymbol{\omega}), D(\mathbf{v}))+2 \alpha \nu \int_{\Gamma_{3}} \boldsymbol{\omega} \cdot \mathbf{v} d \Gamma+(\widetilde{\mathbf{u}} \cdot \nabla \boldsymbol{\omega}, \mathbf{v}) \\
& \quad+(\boldsymbol{\omega} \cdot \nabla \widetilde{\mathbf{u}}, \mathbf{v})+\beta(\mathbf{G} \psi, \mathbf{v})=0, \\
& \kappa(\nabla \psi, \nabla z)+(\widetilde{\mathbf{u}} \cdot \nabla \psi, z)+(\boldsymbol{\omega} \cdot \nabla \widetilde{T}, z) \\
& \quad+\kappa \int_{\Gamma_{5}} \gamma \psi \cdot z d \Gamma=0, \\
& \boldsymbol{\omega}_{|\Gamma| \Gamma_{3}}=\mathscr{B} \boldsymbol{\varrho}, \\
& \psi_{\mid \Gamma_{4}}=\tau,
\end{aligned}
$$

for all $(\mathbf{v}, z) \in \mathbb{X}$. Thus, by using Lemma 3 , there exists $\left(\boldsymbol{\omega}^{\varepsilon}, \psi^{\varepsilon}\right) \in \mathbb{U}$ such that $\boldsymbol{\omega}_{|\Gamma| \Gamma_{3}}^{\varepsilon}=\mathscr{B} \boldsymbol{Q}, \boldsymbol{\omega}_{\mid \Gamma_{3}}=\mathbf{0}, \psi_{\mid \Gamma_{4}}=\tau$, and $\psi_{\mid \Gamma_{5}}=0$. Then, rewriting $(\boldsymbol{\omega}, \psi) \in \mathbb{U}$ in the form $\boldsymbol{\omega}=\boldsymbol{\omega}^{\varepsilon}+\widehat{\boldsymbol{\omega}}$ and $\psi=\psi^{\varepsilon}+\widehat{\psi}$ with $(\widehat{\boldsymbol{\omega}}, \widehat{\psi}) \in \mathbb{X}$ new unknown, and similarly, as in the proof of Lemma 14, we obtain

$$
a((\widehat{\boldsymbol{\omega}}, \widehat{\psi}),(\mathbf{v}, z))=\widetilde{\mathscr{F}}(\mathbf{v}, z) \quad \forall(\mathbf{v}, z) \in \mathbb{X},
$$


where the form $a(\cdot, \cdot)$ is defined in (66) and $\widetilde{\mathscr{F}} \in \mathbb{X}^{\prime}$ is defined by $\widetilde{\mathscr{F}}(\mathbf{v}, z):=\langle\widetilde{\mathbf{a}}, \mathbf{v}\rangle_{\widetilde{\mathbf{H}}_{\sigma}^{1}}+\langle\widetilde{b}, z\rangle_{H^{\prime}}$, with $\langle\widetilde{\mathbf{a}}, \mathbf{v}\rangle_{\widetilde{\mathbf{H}}_{\sigma}^{\prime}}:=$ $-2 \nu\left(D\left(\boldsymbol{\omega}^{\varepsilon}\right), D(\mathbf{v})\right)-\left(\widetilde{\mathbf{u}} \cdot \nabla \boldsymbol{\omega}^{\varepsilon}, \mathbf{v}\right)-\left(\boldsymbol{\omega}^{\varepsilon} \cdot \nabla \widetilde{\mathbf{u}}, \mathbf{v}\right)-\beta\left(\mathbf{G} \psi^{\varepsilon}, \mathbf{v}\right)$ and $\langle\widetilde{b}, z\rangle_{H^{\prime}}:=-\kappa\left(\nabla \psi^{\varepsilon}, \nabla z\right)-\left(\widetilde{\mathbf{u}} \cdot \nabla \psi^{\varepsilon}, z\right)-\left(\boldsymbol{\omega}^{\varepsilon} \cdot \nabla \widetilde{T}, z\right)$. Also, from (100), we can obtain the fact that there exists a positive constant $C$ depending only on $\nu, \kappa$ and $\|(\widetilde{\mathbf{u}}, \widetilde{T})\|_{\circledast}$, such that $\|(\widehat{\boldsymbol{\omega}}, \widehat{\psi})\|_{\mathbb{X}} \leq(C / \widetilde{\delta})\left\|\left(\boldsymbol{\omega}^{\varepsilon}, \psi^{\varepsilon}\right)\right\|_{\sharp}$, where $\widetilde{\delta}$ is given as in (71). Thus, we have

$$
\begin{aligned}
\|(\boldsymbol{\omega}, \psi)\|_{\mathbb{W}} & \leq\|(\widehat{\boldsymbol{\omega}}, \widehat{\psi})\|_{\mathbb{X}}+\left\|\left(\boldsymbol{\omega}^{\varepsilon}, \psi^{\varepsilon}\right)\right\|_{\mathbb{H}} \\
& \leq C \frac{(1+\widetilde{\delta})}{\widetilde{\delta}}\left(\|\boldsymbol{\rho}\|_{\mathbf{H}^{1 / 2}\left(\Gamma_{2}\right)}+\|\tau\|_{H^{1 / 2}\left(\Gamma_{4}\right)}\right) .
\end{aligned}
$$

Using (101) in (98) and taking into account the fact that $\Pi:=$ $\min \left\{C b_{1} \widehat{\delta}^{2} /(1+\widehat{\delta})^{2}, C b_{2} \widehat{\delta}^{2} /(1+\widehat{\delta})^{2}\right\}$, we obtain

$$
\begin{aligned}
& \mathscr{L}_{\mathbf{s s}}(\widetilde{\mathbf{s}}, \widetilde{\phi})(\mathbf{t}, \mathbf{t}) \\
& \geq\|\boldsymbol{\omega}\|^{2}+\|\psi\|^{2}+\frac{b_{1}}{2}\|\boldsymbol{\varrho}\|_{\mathbf{H}^{1 / 2}\left(\Gamma_{2}\right)}^{2}+\frac{b_{2}}{2}\|\tau\|_{H^{1 / 2}\left(\Gamma_{4}\right)}^{2} \\
& -C\left(\|\lambda\|_{\widetilde{H}_{\sigma}^{1}}+\|\eta\|_{H_{0}}\right)\|(\boldsymbol{\omega}, \psi)\|_{\mathbb{H}}^{2}+\Pi\|(\boldsymbol{\omega}, \psi)\|_{\mathbb{H}}^{2} \\
& \geq\left(\Pi-C\left(\|\lambda\|_{\widetilde{H}_{\sigma}^{1}}+\|\eta\|_{H_{0}}\right)\right)\|(\boldsymbol{\omega}, \psi)\|_{\omega \|}^{2} \\
& +\frac{b_{1}}{2}\|\varrho\|_{\mathbf{H}^{1 / 2}\left(\Gamma_{2}\right)}^{2}+\frac{b_{2}}{2}\|\tau\|_{H^{1 / 2}\left(\Gamma_{4}\right)}^{2} .
\end{aligned}
$$

Notice that, from Lemma 18, we can obtain $\|\lambda\|_{\widetilde{\mathbf{H}}_{\sigma}^{1}}+\|\eta\|_{H_{0}} \leq$ $((\widetilde{C} / \widehat{\delta}) \mathcal{N}(\widetilde{\mathbf{u}}, \widetilde{T}))^{1 / 2}$; then, from (102), we have

$$
\begin{aligned}
& \mathscr{L}_{\mathbf{s s}}(\widetilde{\mathbf{s}}, \widetilde{\phi})(\mathbf{t}, \mathbf{t}) \\
& \geq\left[\Pi-C\left(\frac{\widetilde{C}}{\widehat{\delta}} \mathcal{N}(\widetilde{\mathbf{u}}, \widetilde{T})\right)^{1 / 2}\right]\|(\boldsymbol{\omega}, \psi)\|_{\mathbb{H}}^{2} \\
& \quad+\frac{b_{1}}{2}\|\boldsymbol{l}\|_{\mathbf{H}^{1 / 2}\left(\Gamma_{2}\right)}^{2}+\frac{b_{2}}{2}\|\tau\|_{H^{1 / 2}\left(\Gamma_{4}\right)}^{2} .
\end{aligned}
$$

Since $C((\widetilde{C} / \widehat{\delta}) \mathcal{N}(\widetilde{\mathbf{u}}, \widetilde{T}))^{1 / 2}<\Pi$, then we have $\widetilde{\Pi}:=\Pi-$ $C((\widetilde{C} / \widehat{\delta}) \mathcal{N}(\widetilde{\mathbf{u}}, \widetilde{T}))^{1 / 2}>0$; therefore

$$
\mathscr{L}_{\text {ss }}(\widetilde{\mathbf{s}}, \widetilde{\phi})(\mathbf{t}, \mathbf{t}) \geq \min \left\{\widetilde{\Pi}, \frac{b_{1}}{2}, \frac{b_{2}}{2}\right\}\|\mathbf{t}\|_{\mathbb{W} \times \mathscr{U}_{\mathrm{ad}}}^{2} .
$$

Thus, taking in particular $\mathbf{t}=((\boldsymbol{\omega}, \psi),(\boldsymbol{\varrho}, \tau)) \in \operatorname{ker}\left(\mathbb{F}_{\mathbf{s}}(\widetilde{\mathbf{s}})\right)$ with $(\boldsymbol{\varrho}, \tau) \in \mathscr{C}\left(\widetilde{\mathbf{g}}_{1}\right) \times \mathscr{C}\left(\widetilde{g}_{2}\right):=\left\{\left(\theta_{1}\left(\mathbf{g}_{1}-\widetilde{\mathbf{g}}_{1}\right), \theta_{2}\left(g_{2}-\widetilde{g}_{2}\right)\right): \theta_{1} \geq\right.$ $\left.0, \theta_{2} \geq 0,\left(\mathbf{g}_{1}, g_{2}\right) \in \mathscr{U}_{\mathrm{ad}}\right\}$, we obtain that the point $\widetilde{\mathbf{s}} \in \mathcal{S}_{\mathrm{ad}}$ is a local optimal solution of control problem (45) (see [34]).

\section{Conclusions}

In this paper, the existence and uniqueness of weak solutions of the stationary heat convection equations with mixed boundary conditions, including the Navier slip condition, were proved. Also, an optimal boundary control problem was analyzed. Boundary controls for the velocity vector and temperature and the existence of optimal solutions were proved. The optimal control problem analyzed includes the minimization of the $L^{2}$-distance between the velocities and some desired fields. By using the theorem of Lagrange multipliers, an optimality system was derived. A secondorder sufficient condition was also given.

\section{Conflicts of Interest}

The authors declare that there are no conflicts of interest regarding the publication of this paper.

\section{Acknowledgments}

The first author was supported by Proyecto UTA-Mayor 473817, Universidad de Tarapacá, Chile. The second author was supported by the Area of Exact Basics and the Research Department of the Universidad del Sinú, Colombia.

\section{References}

[1] M. D. Gunzburger, Perpectives in Flow Control and Optimization, SIAM, 2003.

[2] L. S. Hou and T. P. Svobodny, "Optimization Problems for the Navier-Stokes Equations with Regular Boundary Controls," Journal of Mathematical Analysis and Applications, vol. 177, no. 2, pp. 342-367, 1993.

[3] F. Guillén-González and G. Planas, "On the asymptotic behaviour of the 2D Navier-Stokes equations with navier friction conditions towards euler equations," ZAMM: Zeitschrift für Angewandte Mathematik und Mechanik 89, vol. 89, no. 10, pp. 810-822, 2009.

[4] C. L. M. H. Navier, "Sur le lois de léquilibrie et du mouvement des corps élastiques," Mem. and Acad. R. Sci. Inst. France 369, vol. 369, 1827.

[5] J. C. Maxwell, "On stressed in rariffed gases arising from inequalities of temperature," Phil. Trans. Royal Society, pp. 704$712,1879$.

[6] W. Jäger and A. Mikelić, "On the roughness-induced effective boundary conditions for an incompressible viscous flow," Journal of Differential Equations, vol. 170, no. 1, pp. 96-122, 2001.

[7] V. Y. Belov and B. V. Kapitonov, "A certain hydrodynamic model of a chemically active fluid," Siberian Mathematical Journal, vol. 24, no. 6, pp. 823-833, 1983.

[8] M. Gaultier and M. Lezaun, "Équations de Navier-Stokes couplées a des équations de la chaleur: Résolution par une méthode de point fixe en dimension infinie," Annales mathématiques du Québec, vol. 13, pp. 1-17, 1989.

[9] H.-C. Lee and S. Kim, "Finite element approximation and computations of optimal Dirichlet boundary control problems for the Boussinesq equations," Journal of the Korean Mathematical Society, vol. 41, no. 4, pp. 681-715, 2004.

[10] H. Morimoto, "On the existence and uniqueness of the stationary solution to the equations of natural convection," Tokyo Journal of Mathematics, vol. 14, no. 1, pp. 218-226, 1991.

[11] H. Morimoto, "Heat convection equation with nonhomogeneous boundary condition," Funkcialaj Ekvacioj, vol. 53, no. 2, pp. 213-229, 2010.

[12] M. S. Da Rocha, M. A. Rojas-Medar, and M. D. Rojas-Medar, "On the existence and uniqueness of the stationary solution to equations of natural convection with boundary data in L2," 
Proceedings of the Royal Society A Mathematical, Physical and Engineering Sciences, vol. 459, no. 2031, pp. 609-621, 2003.

[13] S. Rionero and G. Mulone, "Existence and uniqueness theorems for a steady thermo-diffusive mixture in a mixed problem," Nonlinear Analysis: Theory, Methods and Applications 12, vol. 12, no. 5, pp. 473-494, 1988.

[14] C. Amrouche, P. Penel, and N. Seloula, "Some Remarks on the Boundary Conditions in the Theory of Navier-Stokes Equations," Annales Mathématiques Blaise Pascal, vol. 20, no. 1, pp. 37-73, 2013.

[15] C. Amrouche and A. Rejaiba, "Lp-theory for Stokes and NavierStokes equations with Navier boundary condition," Journal of Differential Equations, vol. 256, no. 4, pp. 1515-1547, 2014.

[16] H. Beirão da Veiga, "Regularity for stokes and generalized stokes systems under nonhomogeneous slip-type boundary conditions," Advances in Differential Equations, vol. 9, no. 9-10, pp. 1079-1114, 2004.

[17] L. C. F. Ferreira, G. Planas, and E. J. Villamizar-Roa, "On the nonhomogeneous navier-stokes system with navier friction boundary conditions," SIAM Journal on Mathematical Analysis, vol. 45, no. 4, pp. 2576-2595, 2013.

[18] M. C. Lopes Filho, H. J. Nussenzveig Lopes, and G. Planas, "On the inviscid limit for two-dimensional incompressible flow with Navier friction condition," SIAM Journal on Mathematical Analysis, vol. 36, no. 4, pp. 1130-1141, 2005.

[19] V. A. Solonnikov and V. E. Scadilov, "A certain boundary value problem for the stationary system of Navier-Stokes equations," Trudy Matematicheskogo Institut Imeni V.A. Steklova, vol. 125, pp. 196-210, 1973.

[20] E. Mallea-Zepeda, E. Ortega-Torres, and E. J. Villamizar-Roa, "A boundary control problem for micropolar fluids," Journal of Optimization Theory and Applications, vol. 169, no. 2, pp. 349369, 2016.

[21] E. Mallea-Zepeda, E. Ortega-Torres, and É. J. Villamizar-Roa, "An optimal control problem for the steady nonhomogeneous asymmetric fluids," Applied Mathematics \& Optimization, 2017.

[22] G. V. Alekseev, "Solvability of stationary boundary control problems for heat convection equations," Siberian Mathematical Journal, vol. 39, no. 5, pp. 844-858, 1998.

[23] G. Alekseev and D. Tereshko, "Stability of optimal controls for the stationary boussinesq equations," International Journal of Differential Equations, vol. 2011, Article ID 535736, 2011.

[24] G. V. Alekseev and D. A. Tereshko, "Extremum problems of boundary control for steady equations of thermal convection," Journal of Applied Mechanics and Technical Physics, vol. 51, no. 4, pp. 510-520, 2010.

[25] Y. Kim, S. Kim, and H.-C. Lee, "Finite element approximations and computations of optimal control problems for the stationary Boussinesq equations with distributed and Neumann boundary controls," Far East Journal of Applied Mathematics, vol. 20, article no. 1, pp. 61-100, 2005.

[26] H.-C. Lee, "Optimal control problems for the two dimensional Rayleigh-Bénard type convection by a gradient method," Japan Journal of Industrial and Applied Mathematics, vol. 26, no. 1, pp. 93-121, 2009.

[27] H.-C. Lee and O. Y. Imanuvilov, "Analysis of Neumann boundary optimal control problems for the stationary Boussinesq equations including solid media," SIAM Journal on Control and Optimization, vol. 39, no. 2, pp. 457-477, 2000.

[28] D. A. Rueda-G, E. J. Villamizar-Roa, and D. A. Rueda-Gómez, "On the Rayleigh-Binard-Marangoni system and a related optimal control problem," Computers and Mathematics with Applications, vol. 74, no. 12, pp. 2969-2991, 2017.

[29] R. Dautray and J. Lions, Mathematical Analysis and Numerical Methods for Science and Technology, Springer, Heidelberg, Berlin, 2000.

[30] J. C. De Los Reyes and K. Kunisch, "A semi-smooth Newton method for control constrained boundary optimal control of the Navier-Stokes equations," Nonlinear Analysis: Theory, Methods \& Applications, vol. 62, no. 7, pp. 1289-1316, 2005.

[31] C. Abergel and F. Casas, "Some optimal control problems of multistate equations appearing in fluid mechanics," Mathematical Modelling and Numerical Analysis, vol. 27, no. 2, pp. 223-247, 1993.

[32] R. Temam, Navier-Stokes Equations: Theory and Numerical Analysis, AMS Chelsea Publishing, 2001.

[33] J. Zowe and S. Kurcyusz, "Regularity and stability for the mathematical programming problem in Banach spaces," Applied Mathematics \& Optimization, vol. 5, no. 1, pp. 49-62, 1979.

[34] H. Maurer, "First and second order sufficient optimality conditions in mathematical programming and optimal control," in Mathematical Programming at Oberwolfach, vol. 14 of Mathematical Programming Studies, pp. 163-177, Springer, Heidelberg, Berlin, Germany, 1981. 


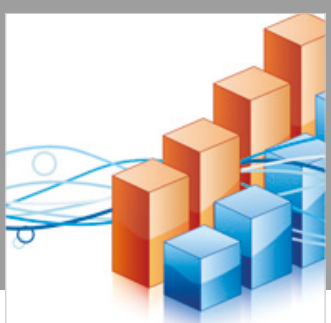

Advances in

Operations Research

\section{-n-m}
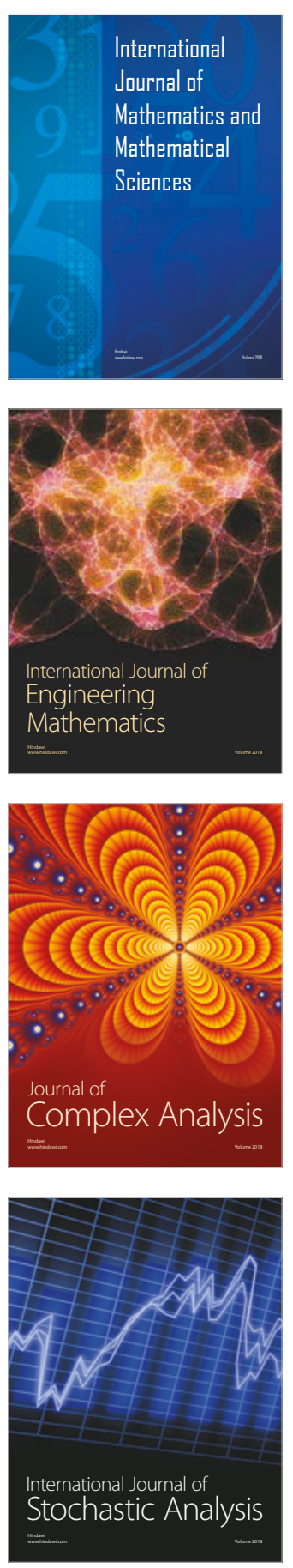
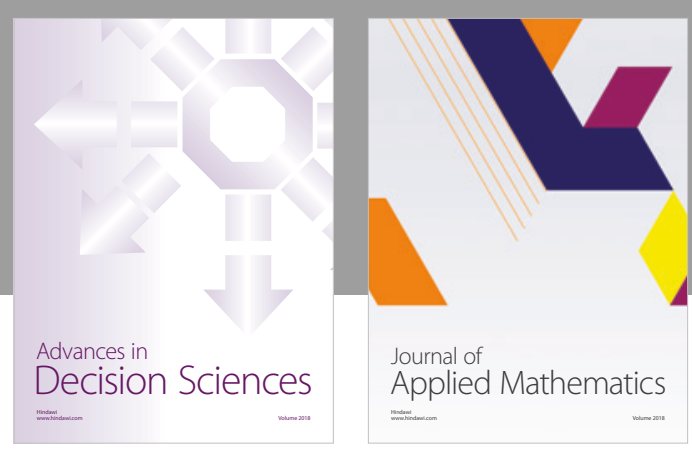

Journal of

Applied Mathematics
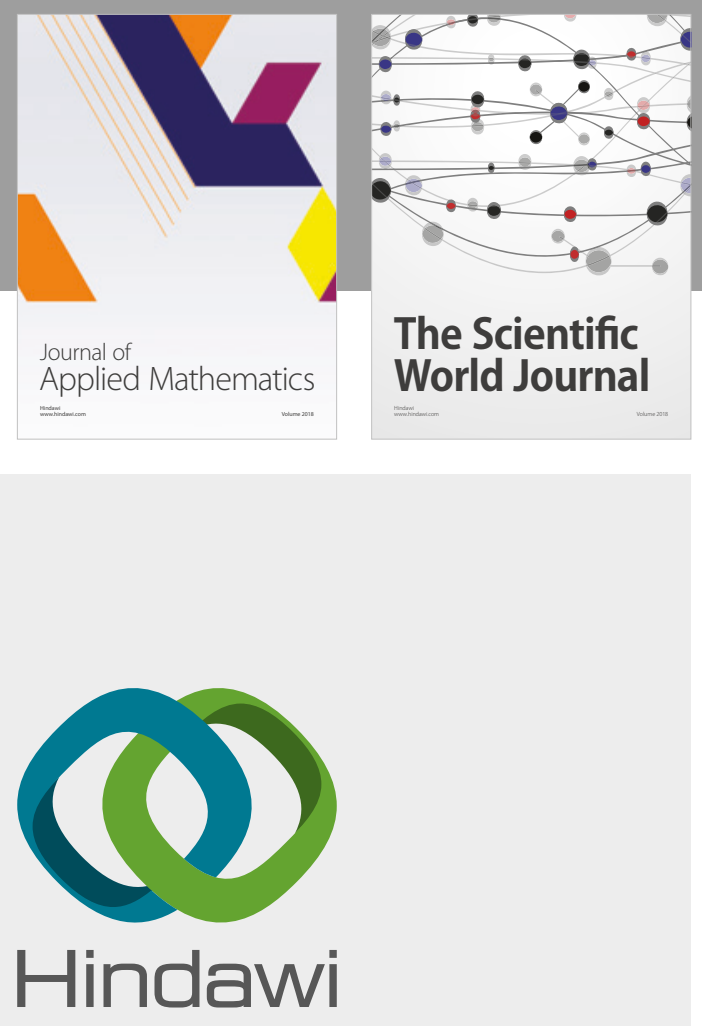

Submit your manuscripts at

www.hindawi.com

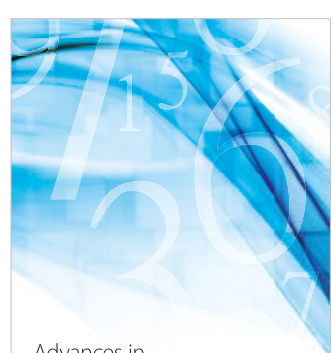

Advances in
Numerical Analysis
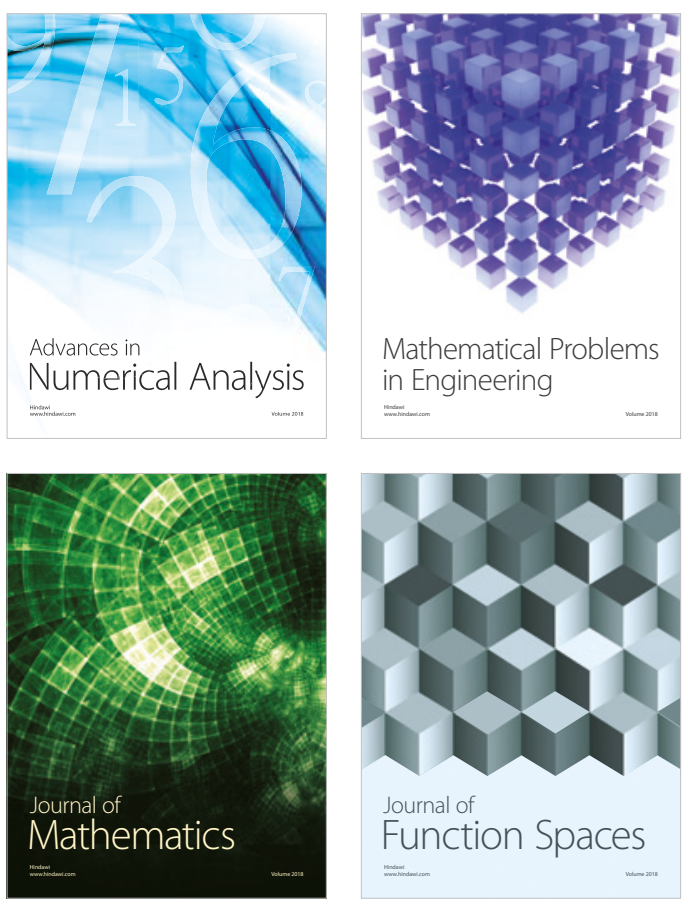

Mathematical Problems in Engineering

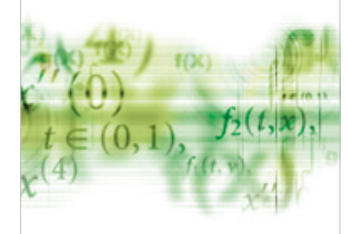

International Journal of

Differential Equations

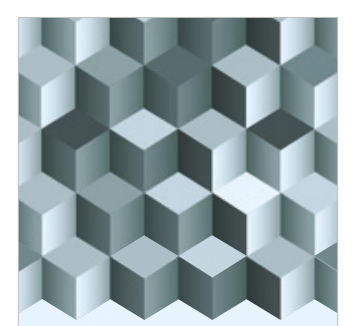

Journal of

Function Spaces

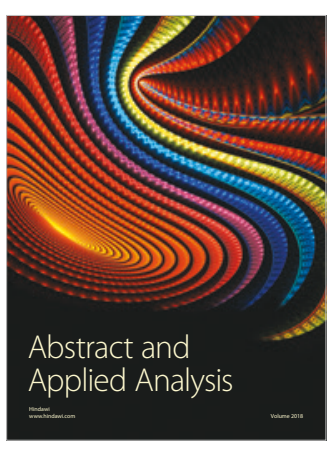

The Scientific

World Journal

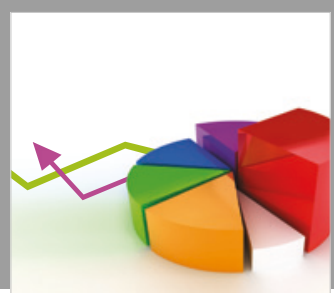

Journal of

Probability and Statistics
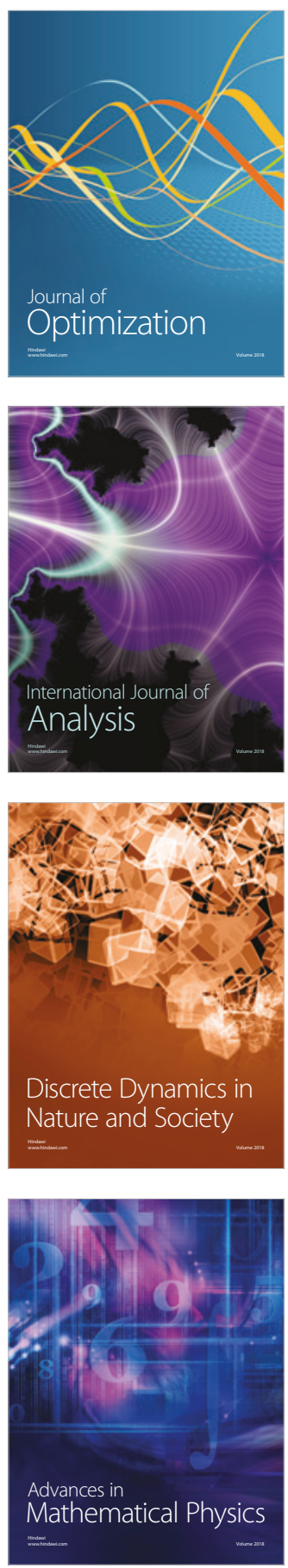